\title{
Pfaffian and Determinantal Tau Functions
}

\author{
JOHAN W. VAN DE LEUR ${ }^{1}$ and ALEXANDER YU. ORLOV ${ }^{2,3}$ \\ ${ }^{1}$ Mathematical Institute, Utrecht University, P.O. Box 80010, 3508 TA Utrecht, \\ The Netherlands.e-mail: J.W.vandeLeur@uu.nl \\ ${ }^{2}$ Institute of Oceanology RAS, Nahimovskii Prospekt 36, Moscow, Russia \\ ${ }^{3}$ International Laboratory of Representation Theory and Mathematical Physics, National \\ Research University Higher School of Economics, 20 Myasnitskaya Ulitsa, Moscow 101000, \\ Russia.e-mail: orlovs@ocean.ru
}

Received: 29 April 2014 / Revised: 14 July 2015 / Accepted: 14 July 2015

Published online: 5 August 2015 - (C) The Author(s) 2015. This article is published with open access at Springerlink.com

\begin{abstract}
Adler, Shiota and van Moerbeke observed that a tau function of the Pfaff lattice is a square root of a tau function of the Toda lattice hierarchy of Ueno and Takasaki. In this paper, we give a representation theoretical explanation for this phenomenon. We consider 2-BKP and two-component 2-KP tau functions. We shall show that a square of a BKP tau function is equal to a certain two-component KP tau function and a square of a 2-BKP tau function is equal to a certain two-component 2-KP tau function.
\end{abstract}

Mathematics Subject Classification. 17B65, 17B80, 35Q51, 35Q53, 35Q55.

Keywords. integrable systems, Tau functions, BKP, DKP, two-component Toda lattice, Pfaff lattice, free fermions.

\section{Introduction}

Sato and Sato [27] and Date, Jimbo, Kashiwara and Miwa [4] introduced and described in the beginning of the 1980s the KP hierarchy in various setting. They introduced a tau function, which is a fundamental object in this theory. It is an element in a $G L_{\infty}$ group orbit and as such a solution of the KP hierarchy. Around the same time Date, Jimbo, Kashiwara and Miwa introduced in [5] also a new hierarchy of soliton equations, which they called the KP hierarchy of type B or BKP hierarchy. The corresponding tau function is an element of the $B_{\infty}$ group orbit and hence a solution to this new BKP hierarchy. In a straightforward calculation, they show that this BKP tau function is the square root of a certain KP tau function. Their proof of this phenomenon is fundamental for the contents of this paper. Here, we show that this method is also applicable for the observation of Adler, Shiota and van Moerbeke [1,2], that a tau function of the Pfaff lattice is a square root of an Ueno, Takasaki [32] Toda lattice tau function. In this introduction, we will recall the work of Date, Jimbo, Kashiwara and Miwa [4] and explain the relation BKP versus KP. The involutions that are used in their work, 
which provide the relation between the two tau functions of KP and BKP, also give the relation between the various tau functions of the Pfaff and Toda lattice. This means that the construction of the KP group element out of the BKP group element is the same. One finds in both cases that $g_{\mathrm{KP}}=h_{\mathrm{BKP}} \hat{h}_{\mathrm{BKP}}$, where $\hat{h}_{\mathrm{BKP}}$ is constructed out of $h_{\mathrm{BKP}}$ using one of the involutions.

The Pfaff lattice of Adler and van Moerbeke [1,2] was discovered by Jimbo and Miwa ([10], section 7) it was rediscovered by Hirota and Ohta [13] as the coupled KP hierarchy and studied in a paper by Kac and one of the authors [15] as the charged DKP hierarchy. The Pfaff lattice studied in [16] is slightly bigger than the one studied by Adler, Shiota and van Moerbeke in [2] it is the charged BKP hierarchy of [15], called the large BKP in [25] to make difference to the small BKP of [5]. Here, we study this charged or large BKP.

In addition, we also consider the large 2-BKP which is two-sided evolution (via positive and negative parts of current modes, like the Toda lattice hierarchy of Ueno and Takasaki [32] in contrast to the one-sided KP). There we have two sets of higher times, $t$ and $\bar{t}$. This is the B-analog of Takasaki's 2-DKP of [29] (which he called the Pfaff lattice by analogy with Pfaff lattice of Adler and van Moerbeke). Also, we use two discrete variables for BKP (and also for 2-BKP): $\tau_{m, n}(t, \bar{t})$.

In this introduction and the first 5 sections we will only describe the one-sided case, to avoid technicalities. In Section 6 we will introduce the two-sided case, which will be important for matrix models.

For matrix models, we need the semi-infinite Toda and Pfaff lattices. The fermionic expressions for the related tau functions is considered in the Appendix A.1 where a set of examples is written down. In this Section, we generalize the observation of Adler, van Moerbeke and Shiota [1,2] about the relation between Toda and Pfaff lattices.

But, first, we will start with the recollection of the work of Date, Jimbo, Kashiwara, and Miwa, on KP and BKP hierarchies.

Fermions and KP hierarchy Consider, following [4] and [10] charged fermions $\left\{f_{i}, f_{i}^{\dagger}\right\}_{i \in \mathbb{Z}}$, satisfying

$$
\left[f_{i}, f_{j}^{\dagger}\right]_{+}=\delta_{i j}, \quad\left[f_{i}, f_{j}\right]_{+}=0=\left[f_{i}^{\dagger}, f_{j}^{\dagger}\right]_{+}, \quad i, j \in \mathbb{Z}
$$

where $[x, y]_{+}=x y+y x$ is anticommutator. The elements $f_{i}$ and $f_{i}^{\dagger}$ form the basis of a vector space, which we denote by $V$. Introduce the spin modules (right and left Fock spaces) with vacuum vectors, $|0\rangle,\langle 0|$, such that

$$
f_{i}|0\rangle=f_{-1-i}^{\dagger}|0\rangle=0=\langle 0| f_{i}^{\dagger}=\langle 0| f_{-1-i}, \quad i<0
$$

Let $g \in G L_{\infty}$ which may be written as

$$
g=\exp \sum_{i, j \in \mathbb{Z}} a_{i j}: f_{i} f_{j}^{\dagger}:
$$


where $: f_{i} f_{j}^{\dagger}$ : denotes $f_{i} f_{j}^{\dagger}-\left\langle 0\left|f_{i} f_{j}^{\dagger}\right| 0\right\rangle$, and $a_{i j}$ are some complex numbers. The elements : $f_{i} f_{j}^{\dagger}$ : together with 1 form a basis of the Lie algebra $g l_{\infty}$, see [4], [10] for more details.

Then,

$$
\sum_{i \in \mathbb{Z}} f_{i} g|0\rangle \otimes f_{i}^{\dagger} g|0\rangle=0
$$

is the KP hierarchy in the fermionic form, see [4].

One turns this equation into a hierarchy of differential equation using the boson-fermion correspondence, see, e.g., [14] for more details.

However, the tau function can be be calculated in a different way, define the oscillator algebra

$$
V=\left\{\alpha_{n}=\sum_{i \in \mathbb{Z}}: f_{i} f_{i+n}^{\dagger}:\right\}
$$

We have

$$
\left[\alpha_{n}, \alpha_{m}\right]_{-}=n \delta_{n+m, 0}
$$

where $[x, y]_{-}=x y-y x$ is commutator. Define

$$
f(z)=\sum_{i \in \mathbb{Z}} f_{i} z^{i}, \quad f^{\dagger}(z)=\sum_{i \in \mathbb{Z}} f_{i}^{\dagger} z^{-i}
$$

then,

$$
\left[\alpha_{k}, f(z)\right]=z^{k} f(z) \quad \text { and }\left[\alpha_{k}, f^{\dagger}(z)\right]=-z^{k} f^{\dagger}(z)
$$

Now,

$$
\alpha_{n}|0\rangle=\langle 0| \alpha_{-n}=0, \quad n \geq 0 .
$$

Then $\tau_{\mathrm{KP}}(t)$, defined by the following expectation value

$$
\tau_{\mathrm{KP}}(t)=\left\langle 0\left|\exp \left(\sum_{k=1}^{\infty} t_{k} \alpha_{k}\right) g\right| 0\right\rangle,
$$

is a solution of the KP hierarchy.

Neutral fermions and the BKP hierarchy. Following [10], we introduce an involution $\omega$ on the Clifford algebra, defined by

$$
\omega\left(f_{i}\right)=(-)^{i} f_{-i}^{\dagger}, \quad \omega\left(f_{i}^{\dagger}\right)=(-)^{i} f_{-i}
$$

The fixed points of $\omega$ in the vector space $V$ with basis $f_{i}, f_{i}^{\dagger}$ are the elements

$$
b_{j}=\frac{f_{j}+(-)^{j} f_{-j}^{\dagger}}{\sqrt{2}} .
$$


The elements

$$
\hat{b}_{j}=i \frac{f_{j}-(-)^{j} f_{-j}^{\dagger}}{\sqrt{2}}
$$

are elements in the -1 eigenspace of $\omega . b_{j}$ and $\hat{b}_{j}$ form a new basis of $V$, we have

$$
\left[b_{i}, \hat{b}_{j}\right]=0, \quad\left[b_{i}, b_{j}\right]=\left[\hat{b}_{i}, \hat{b}_{j}\right]=(-)^{i} \delta_{i,-j}
$$

The fixed points in $g l_{\infty}$ are 1 and the elements

$$
(-)^{k}: f_{j} f_{-k}^{\dagger}:-(-)^{j}: f_{k} f_{-j}^{\dagger}:
$$

These elements form a Lie algebra of type $b_{\infty}$. If one considers the action of these elements on the vacua of the spin modules, one obtains a level two representation of $b_{\infty}$ (Note that here we allow also certain infinite sums of these elements).

Note also that only the $\alpha_{k}$ for $k$ odd are fixed by $\omega$.

It is straightforward to check that

$$
(-)^{k}: f_{j} f_{-k}^{\dagger}:-(-)^{j}: f_{k} f_{-j}^{\dagger}:=: b_{j} b_{k}:+: \hat{b}_{j} \hat{b}_{k}: .
$$

The following observation is crucial. The elements $: b_{j} b_{k}$ : together with 1 , or the elements $: \hat{b}_{j} \hat{b}_{k}$ : together with 1 , separately form the Lie algebra $b_{\infty}$. These two Lie algebras that have $\mathbb{C} 1$ as intersection, commute. If one considers the action of these elements separately on the vacua, one obtains a level one representation. The corresponding module in terms of the $b_{j}$ or $\hat{b}_{j}$ is called the spin module of type B. One has

$$
b_{j}|0\rangle=\hat{b}_{j}|0\rangle=\langle 0| b_{-j}=\langle 0| \hat{b}_{-j}, \quad j<0 .
$$

and

$$
b_{0}|0\rangle=-i \hat{b}_{0}|0\rangle=\frac{1}{2} \sqrt{2} f_{0}|0\rangle, \quad \text { and }\langle 0| b_{0}=i\langle 0| \hat{b}_{0}=\frac{1}{2} \sqrt{2}\langle 0| f_{0}^{\dagger},
$$

hence

$$
\left\langle 0\left|b_{0} b_{0}\right| 0\right\rangle=-i\left\langle 0\left|b_{0} \hat{b}_{0}\right| 0\right\rangle=i\left\langle 0\left|\hat{b}_{0} b_{0}\right| 0\right\rangle=\left\langle 0\left|\hat{b}_{0} \hat{b}_{0}\right| 0\right\rangle=\frac{1}{2} .
$$

As in the KP case we now consider an element in the group $h \in B_{\infty}$, such an element may, e.g., be written as

$$
h=\exp \sum_{i, j \in \mathbb{Z}} a_{i j}: b_{i} b_{j}:
$$

Then,

$$
\sum_{i \in \mathbb{Z}}(-)^{i} b_{i} h|0\rangle \otimes b_{-i} h|0\rangle=h b_{0}|0\rangle \otimes h b_{0}|0\rangle
$$


is the BKP hierarchy in the fermionic form, see, e.g., [10]. One turns this into a hierarchy of differential equations using twisted vertex operators, see [15] for more details. The corresponding BKP tau function can be obtained as follows. Define the twisted oscillator algebra

$$
\gamma_{n}=\frac{1}{2} \sum_{i \in \mathbb{Z}}(-)^{i+1}: b_{i} b_{-i-n}:
$$

Then, $\gamma_{n}=0$ if $n$ is even. We have

$$
\left[\gamma_{n}, \gamma_{m}\right]_{-}=\frac{n}{2} \delta_{n+m, 0}
$$

and

$$
\gamma_{n}|0\rangle=\langle 0| \gamma_{-n}=0, \quad n \geq 0 .
$$

Let

$$
b(z)=\sum_{i \in \mathbb{Z}} b_{i} z^{i},
$$

then,

$$
\left[\gamma_{k}, b(z)\right]=z^{k} b(z)
$$

The BKP tau function $\tau_{\mathrm{BKP}}(t)$ is defined by the following expectation value

$$
\tau_{\mathrm{BKP}}(t)=\left\langle 0\left|\exp \left(\sum_{k=1, \text { odd }}^{\infty} t_{k} \gamma_{k}\right) h\right| 0\right\rangle
$$

and is a solution of the BKP hierarchy. A crucial observation is the fact that in this construction we could have replaced the $b_{n}$ by $\hat{b}_{n}$ and in this way would have obtained the same result.

A relation between KP and BKP tau functions We follow Jimbo and Miwa [10] or rather You [34] and define an isomorphism ^ on the B-type Clifford algebra

$$
\wedge^{\wedge}\left(b_{n}\right)=\hat{b}_{n} .
$$

Now,

$$
: b_{n} b_{m}: \hat{+}^{\wedge}\left(: b_{n} b_{m}:\right)=: b_{n} b_{m}:+: \hat{b}_{n} \hat{b}_{m}:=(-)^{m}: f_{n} f_{-m}^{\dagger}:-(-)^{n}: f_{m} f_{-n}^{\dagger}:
$$

and

$$
\gamma_{n} \hat{+}^{\wedge}\left(\gamma_{n}\right)=\gamma_{n}+\hat{\gamma}_{n}=\alpha_{n}, \quad n \text { odd }
$$

Let $g=h \hat{h}$, where ${ }^{\wedge}=\hat{h}$ and $h \in B_{\infty}$, then $h$ and $\hat{h}$ commute, Now consider the expression 


$$
\begin{aligned}
\tau(t, \hat{t}) & =\left\langle 0\left|\exp \left(\sum_{k=1, \text { odd }}^{\infty}\left(t_{k} \gamma_{k}+\hat{t}_{k} \hat{\gamma}_{k}\right)\right) h \hat{h}\right| 0\right\rangle \\
& =\left\langle 0\left|\exp \left(\sum_{k=1, \text { odd }}^{\infty} t_{k} \gamma_{k}\right) h \exp \left(\sum_{k=1, \text { odd }}^{\infty} \hat{t}_{k} \hat{\gamma}_{k}\right) \hat{h}\right| 0\right\rangle,
\end{aligned}
$$

where $t_{k}$ and $\hat{t}_{k}$ are two sets of parameters, the times of the BKP hierarchy. The above expression (31) can be reduced, i.e., one can move the exponentials to the right such that they act as 1 on the right vacuum $|0\rangle$. Since $\gamma_{k}$ commutes with $\hat{h}$ and $\hat{\gamma}_{k}$ commutes with $h$ and the action of $\gamma_{k}$ on $h$ is the same as $\hat{\gamma}_{k}$ on $\hat{h}$ (because $\left[\gamma_{k}, b_{\ell}\right]=b_{\ell-k}$ and $\left.\left[\hat{\gamma}_{k}, \hat{b}_{\ell}\right]=\hat{b}_{\ell-k}\right)$, we find that

$$
\tau(t, \hat{t})=\left\langle 0\left|\left(\sum_{I} S_{I}(t) \mathbf{b}_{I}\right)\left(\sum_{I} S_{I}(\hat{t}) \hat{\mathbf{b}}_{I}\right)\right| 0\right\rangle
$$

where $\mathbf{b}_{I}=b_{i_{2 k}} b_{i_{2 k-1}} \ldots b_{i_{1}}$ with $i_{2 k}>i_{2 k-1}>\cdots>i_{1}$ and $\hat{\mathbf{b}}_{I}$ is the same element but then with the $b_{j}$ s replaced by $\hat{b}_{j}$ s. Using the action on the left and right vacuum of the elements, we find that

$$
\tau(t, \hat{t})=S_{\emptyset}(t) S_{\emptyset}(\hat{t})
$$

Now note that also

$$
S_{\emptyset}(t)=\left\langle 0\left|\sum_{I} S_{I}(t) \mathbf{b}_{I}\right| 0\right\rangle=\left\langle 0\left|\exp \left(\sum_{k=1, \text { odd }}^{\infty} t_{k} \gamma_{k}\right) h\right| 0\right\rangle
$$

hence

$$
\begin{aligned}
\left(\tau_{\mathrm{BKP}}(t)\right)^{2} & =\left\langle 0\left|\exp \left(\sum_{k=1, \text { odd }}^{\infty} t_{k} \gamma_{k}\right) h\right| 0\right\rangle\left\langle 0\left|\exp \left(\sum_{k=1, \text { odd }}^{\infty} t_{k} \hat{\gamma}_{k}\right) \hat{h}\right| 0\right\rangle \\
& =\left\langle 0\left|\exp \left(\sum_{k=1, \text { odd }}^{\infty} t_{k}\left(\gamma_{k}+\hat{\gamma}_{k}\right)\right) h \hat{h}\right| 0\right\rangle
\end{aligned}
$$

Now using (30), we find

$$
\left(\tau_{\mathrm{BKP}}(t)\right)^{2}=\left\langle 0\left|\exp \left(\sum_{k=1, \text { odd }}^{\infty} t_{k} \alpha_{k}\right) h \hat{h}\right| 0\right\rangle=\tau_{\mathrm{KP}}\left(t_{1}, 0, t_{3}, 0, t_{5}, 0, \ldots\right),
$$

for $g=h \hat{h}$. Since $h$ is of the form (20) we find, using (29), that

$$
\begin{aligned}
g=h \hat{h} & =\exp \sum_{n, m \in \mathbb{Z}} a_{n m}\left(: b_{n} b_{m}:+: \hat{b}_{n} \hat{b}_{m}:\right) \\
& =\exp \sum_{n, m \in \mathbb{Z}} a_{n m}\left((-)^{m}: f_{n} f_{-m}^{\dagger}:-(-)^{n}: f_{m} f_{-n}^{\dagger}:\right) .
\end{aligned}
$$


The element $(-)^{m}: f_{n} f_{-m}^{\dagger}:-(-)^{n}: f_{m} f_{-n}^{\dagger}$ : is fixed by $\omega$, hence $g \in G L_{\infty}$ is an element in the level two representation of $b_{\infty}$, which one gets by considering the action of the $\omega$ invariant elements in $g l_{\infty}$. In other words, this $\tau_{\mathrm{KP}}\left(t_{1}, 0, t_{3}, 0, t_{5}, 0, \ldots\right)$ is an element in the $B_{\infty}$ group orbit of the vacuum, where we consider $B_{\infty}$ as a subgroup of $G L_{\infty}$ and not as a group acting on the spin module of type $\mathrm{B}$. The latter one is related to the level 1 representation of $b_{\infty}$.

A relation between KP and BKP wave functions Recall from (7) the formula for $f(z)$, the $\mathrm{KP}$ wave function is defined as $W_{\mathrm{KP}}(t, z)=\frac{w_{\mathrm{KP}}(t, z)}{\tau_{\mathrm{KP}}(t)}$, with

$$
w_{\mathrm{KP}}(t, z)=\left\langle 0\left|f_{0}^{\dagger} \exp \left(\sum_{k=1}^{\infty} t_{k} \alpha_{k}\right) f(z) g\right| 0\right\rangle .
$$

Using the boson-fermion correspondence (see, e.g., $[4,14]$ or $(67)$ in the next section),

$$
f(z)=Q z^{\alpha_{0}} e^{-\sum_{k<0}-\frac{\alpha_{k}}{k}} z^{-k} e^{-\sum_{k>0} \frac{\alpha_{k}}{k}} z^{-k},
$$

which is based on (8), then gives

$$
\begin{aligned}
w_{\mathrm{KP}}(t, z) & =e^{\sum_{k>0} t_{k} z^{k}}\left\langle 0\left|\exp \left(\sum_{k=1}^{\infty}\left(t_{k}-\frac{z^{-k}}{k}\right) \alpha_{k}\right) g\right| 0\right\rangle \\
& =\tau_{\mathrm{KP}}\left(t_{1}-\frac{z^{-1}}{1}, t_{2}-\frac{z^{-2}}{2}, t_{3}-\frac{z^{-3}}{3}, \ldots\right) e^{\sum_{k>0} t_{k} z^{k}}
\end{aligned}
$$

Recall $b(z)$ from (25) and define analogously $\hat{b}(z)=\sum_{j \in \mathbb{Z}} \hat{b}_{j} z^{j}$, then using (12) and (13)

$$
f(z)=\frac{1}{\sqrt{2}}(b(z)-i \hat{b}(z)) \quad \text { and } f^{\dagger}(z)=\frac{1}{\sqrt{2}}(b(-z)+i \hat{b}(-z))
$$

Now let, as before, $g=h \hat{h}$, then

$$
\begin{aligned}
w_{\mathrm{KP}}\left(t_{\mathrm{odd}}, z\right) & =w_{\mathrm{KP}}\left(t_{1}, 0, t_{3}, 0, \ldots, z\right) \\
& =\tau_{\mathrm{KP}}\left(t_{1}-\frac{z^{-1}}{1},-\frac{z^{-2}}{2}, t_{3}-\frac{z^{-3}}{3},-\frac{z^{-4}}{4}, \ldots\right) e^{\sum_{k>0} \text { odd } t_{k} z^{k}}
\end{aligned}
$$

Using (26) and the known bosonization formula (6.5) of [10],

$$
\langle 0| b_{0} b(z) e^{\sum_{k=1, \text { odd }} \gamma_{k} t_{k}}=\frac{1}{2}\langle 0| e^{\sum_{k=1, \text { odd }} \gamma_{k}\left(t_{k}-\frac{2 z-k}{k}\right)}
$$


and a similar formula with "hats" ((7.4) in [10]), we obtain

$$
\begin{aligned}
w_{\mathrm{KP}}\left(t_{\mathrm{odd}}, z\right)= & \frac{1}{2}\left\langle 0\left|\left(b_{0}+i \hat{b}_{0}\right) \exp \left(\sum_{k=1, \text { odd }}^{\infty} t_{k}\left(\gamma_{k}+\hat{\gamma}_{k}\right)\right)(b(z)-i \hat{b}(z)) h \hat{h}\right| 0\right\rangle \\
= & \frac{1}{2} e^{\sum_{k>0} \text { odd } t_{k} z^{k}}\left\langle 0\left|\exp \left(\sum_{k=1 \text {, odd }}^{\infty}\left(t_{k}-2 \frac{z^{-k}}{k}\right) \gamma_{k}\right) h\right| 0\right\rangle \\
& \times\left\langle 0\left|\exp \left(\sum_{k=1, \text { odd }}^{\infty} t_{k} \hat{\gamma}_{k}\right) \hat{h}\right| 0\right\rangle \\
& +\frac{1}{2} e^{\sum_{k>0} \text { odd } t_{k} z^{k}}\left\langle 0\left|\exp \left(\sum_{k=1, \text { odd }}^{\infty} t_{k} \gamma_{k}\right) h\right| 0\right\rangle \\
& \times\left\langle 0\left|\exp \left(\sum_{k=1, \text { odd }}^{\infty}\left(t_{k}-2 \frac{z^{-k}}{k}\right) \hat{\gamma}_{k}\right) \hat{h}\right| 0\right\rangle \\
= & \tau_{\mathrm{BKP}}(t) \tau_{\mathrm{BKP}}\left(t_{1}-2 \frac{z^{-1}}{1}, t_{3}-2 \frac{z^{-3}}{3}, \ldots\right) e^{\sum_{k>0} \text { odd } t_{k} z^{k}} .
\end{aligned}
$$

If we now divide this by

$$
\tau_{\mathrm{KP}}\left(t_{1}, 0, t_{3}, 0, \ldots\right)=\left(\tau_{\mathrm{BKP}}(t)\right)^{2},
$$

we obtain an expression for $W_{\mathrm{KP}}\left(t_{\mathrm{odd}}, z\right)$. Define

$$
W_{\mathrm{BKP}}(t, z)=2 \frac{\left\langle 0\left|b_{0} e^{\sum_{k=1, \text { odd }} t_{k} \gamma_{k}} b(z) h\right| 0\right\rangle}{\left\langle 0\left|e^{\sum_{k=1, \text { odd }} t_{k} \gamma_{k}} h\right| 0\right\rangle}=e^{\sum_{k>0, \text { odd }} t_{k} z^{k}}\left(1+O\left(z^{-1}\right)\right) .
$$

Again using (26), one deduces

$$
W_{\mathrm{BKP}}(t, z)=\tau_{\mathrm{BKP}}\left(t_{1}-2 \frac{z^{-1}}{1}, t_{3}-2 \frac{z^{-3}}{3}, \ldots\right) e^{\sum_{k>0} \text { odd } t_{k} z^{k}} / \tau_{\mathrm{BKP}}(t)
$$

Now comparing (44) and (46), we obtain:

$$
W_{\mathrm{BKP}}(t, z)=W_{\mathrm{KP}}\left(t_{1}, 0, t_{3}, 0, \ldots, z\right) .
$$

\section{Another Realization of the KP Hierarchy}

To describe the relation between the Toda lattice hierarchy (or two-component KP) and the Pfaff lattice (or large BKP), we need another realization of both spin modules. We start with the KP hierarchy and relabel the fermions $f_{i}$ and $f_{i}^{\dagger}$ as follows

$$
\begin{aligned}
f_{0} & =\psi, \quad f_{0}^{\dagger}=\psi^{\dagger} \\
f_{2 i+1} & =\psi_{i}^{(1)}, \quad f_{2 i+1}^{\dagger}=\psi_{i}^{\dagger(1)}, \quad i \geq 0
\end{aligned}
$$


while

$$
\begin{aligned}
f_{2 i+2} & =\psi_{i}^{(2)}, \quad f_{2 i+2}^{\dagger}=\psi_{i}^{\dagger(2)}, \quad i \geq 0 \\
f_{2 i} & =\psi_{i}^{(1)}, \quad f_{2 i}^{\dagger}=\psi_{i}^{\dagger(1)}, \quad i<0 \\
f_{2 i+1} & =-\psi_{i}^{(2)}, \quad f_{2 i+1}^{\dagger}=-\psi_{i}^{\dagger(2)}, \quad i<0 .
\end{aligned}
$$

The minus sign in (52) will be convenient later on when we apply the involution $\omega$ to this new realization. Then, for $a, b=1,2$

$$
\left[\psi_{i}^{(a)}, \psi_{j}^{\dagger(b)}\right]_{+}=\delta_{a, b} \delta_{i, j}, \quad \text { and } \quad\left[\psi, \psi^{\dagger}\right]_{+}=1,
$$

all other anti-commutation relations are zero. The action on the vacuum is given by

$$
\begin{aligned}
\psi^{\dagger}|0\rangle & =0=\langle 0| \psi \\
\psi_{i}^{(a)}|0\rangle & =\psi_{-1-i}^{\dagger(\alpha)}|0\rangle=0=\langle 0| \psi_{i}^{\dagger(\alpha)}=\langle 0| \psi_{-1-i}^{(a)}, \quad i<0
\end{aligned}
$$

Now, the KP equation (4) may be rewritten as

$$
\psi g|0\rangle \otimes \psi^{\dagger} g|0\rangle+\sum_{a=1,2} \sum_{i \in \mathbb{Z}} \psi_{i}^{(a)} g|0\rangle \otimes \psi_{i}^{\dagger(\alpha)} g|0\rangle=0
$$

where $g$ is the same as in (4) rewritten as

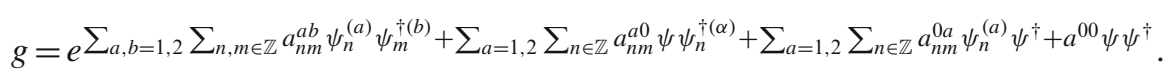

Remark 1. One can identify Equation (56) with the Hirota equation for the threecomponent KP hierarchy $[4,10,14]$ restricted on the class of $g$ which does not depend on $\psi_{k}^{(3)}, \psi_{k}^{\dagger(3)}$ for all $k \neq i$ except on $\psi_{i}^{(3)}:=\psi, \psi_{i}^{\dagger(3)}:=\psi^{\dagger}$ where $i$ is arbitrarily chosen.

It may also be written in the form

$$
g=g^{(0)}+g^{(1)} \psi+g^{(-1)} \psi^{\dagger}, \quad g^{(0)}=g^{(0,0)}+g^{(1,-1)} \psi \psi^{\dagger}
$$

where $g^{(0,0)}, g^{(1,-1)}, g^{(1)}$ and $g^{(-1)}$ do not contain neither $\psi$, nor $\psi^{\dagger}$. Then, $g^{(0)}$ is even and $g^{( \pm 1)}$ are odd in the $Z_{2}$ grading of the Clifford algebra.

Define a two-component oscillator algebra (cf (5))

$$
\alpha_{n}^{(a)}=\sum_{i \in \mathbb{Z}}: \psi_{i}^{(a)} \psi_{i+n}^{\dagger(a)}:
$$

We have

$$
\left[\alpha_{n}^{(a)}, \alpha_{m}^{(b)}\right]_{-}=n \delta_{a, b} \delta_{n+m, 0}
$$


Introduce

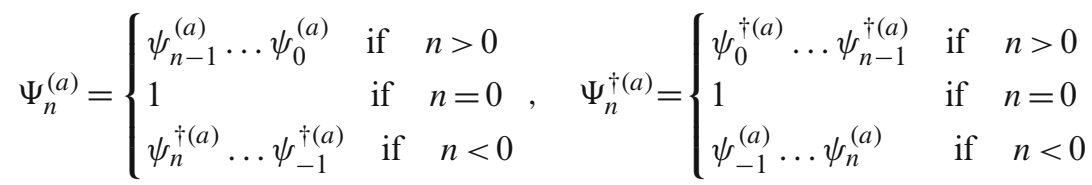

and introduce right and left Fock vectors

$$
\begin{aligned}
& |n, m, 0\rangle=\Psi_{m}^{(2)} \Psi_{n}^{(1)}|0\rangle, \quad\langle n, m, 0|=\langle 0| \Psi_{n}^{\dagger(1)} \Psi_{m}^{\dagger(2)} \\
& |n, m, 1\rangle=\Psi_{m}^{(2)} \Psi_{n}^{(1)} \psi|0\rangle, \quad\langle n, m, 1|=\langle 0| \psi^{\dagger} \Psi_{n}^{\dagger(1)} \Psi_{m}^{\dagger(2)}
\end{aligned}
$$

which are highest weight vectors for the oscillator algebra (59):

$$
\alpha_{i}^{(a)}|n, m, k\rangle=0 \quad \text { if } \quad i>0, \quad\langle n, m, k| \alpha_{-i}^{(a)}=0 \quad \text { if } \quad i>0
$$

for any $a, n, m$ and $k=0,1$.

We have the orthogonality condition $\left\langle n, m, k|1| n^{\prime}, m^{\prime}, k^{\prime}\right\rangle=\delta_{n, n^{\prime}} \delta_{m, m^{\prime}} \delta_{k, k^{\prime}}$ where $n, n^{\prime}, m, m^{\prime} \in \mathbb{Z}, k, k^{\prime}=0,1$.

Now, we can write

$$
g|0\rangle=\sum_{n \in \mathbb{Z}}\left(g_{-n}^{(0)}|n,-n, 0\rangle+g_{-n}^{(1)}|n-1,-n, 1\rangle\right)
$$

Boson-fermion correspondence. Now, we set the following correspondence. The left Fock space is isomorphic to $\left[\theta, q_{a}, q_{a}^{-1}, t_{i}^{(a)} ; a=1,2, i=1,2, \ldots\right]$. Let $\sigma$ be the corresponding isomorphism. Introduce the fermionic fields

$$
\psi^{(a)}(z)=\sum_{i \in \mathbb{Z}} \psi_{i}^{(a)} z^{i}, \quad \psi^{\dagger(a)}(z)=\sum_{i \in \mathbb{Z}} \psi_{i}^{\dagger(a)} z^{-i-1},
$$

then,

$$
\sigma(\psi)=\theta, \quad \sigma\left(\psi^{\dagger}\right)=\frac{\partial}{\partial \theta}
$$

where $\theta^{2}=0$, and

$$
\sigma\left(\psi^{(a)}(z)\right)=q_{a} z^{q_{a} \frac{\partial}{\partial q_{a}}} E^{+(a)}(z), \quad \sigma\left(\psi^{\dagger(a)}(z)\right)=q_{a}^{-1} z^{-q_{a} \frac{\partial}{\partial q_{a}}} E^{-(a)}(z)
$$

where

$$
E^{ \pm(a)}(z)=e^{ \pm \sum_{n=1}^{\infty} t_{n}^{(a)} z^{n}} e^{\mp \sum_{k=1}^{\infty} \frac{1}{k} \frac{\partial}{\partial t_{n}^{(a)}}}
$$

Here,

$$
q_{a} q_{b}=-q_{b} q_{a}, \quad \theta q_{a}=-q_{a} \theta
$$

for $a, b=1,2$ and $a \neq b$. 
Then, (65) turns out to be

$$
\sigma(g|0\rangle)=\sum_{n}\left(\tau_{-n}^{(0)} q_{2}^{n} q_{1}^{-n}+\tau_{-n}^{(1)} q_{2}^{n} q_{1}^{-n-1} \theta\right)
$$

Now, the Hirota KP Equation (4) (rewritten in form (56)) are

$$
\begin{aligned}
& 0=-\sum_{n, m} \tau_{-n}^{(0)} q_{2}^{n} q_{1}^{-n} \theta \otimes \tau_{-m}^{(1)} q_{2}^{m} q_{1}^{-m-1} \\
& +\underset{z}{\operatorname{res}}(-)^{n} z^{-n} E^{+(1)}(z)\left(\tau_{-n}^{(0)} q_{2}^{n} q_{1}^{1-n}+z^{-1} \tau_{-n}^{(1)} q_{2}^{n} q_{1}^{-n} \theta\right) \\
& \otimes(-)^{m} z^{-m} E^{-(1)}(z)\left(\tau_{-m}^{(0)} q_{2}^{m} q_{1}^{-1-m}+z \tau_{-m}^{(1)} q_{2}^{m} q_{1}^{-m-2} \theta\right)
\end{aligned}
$$

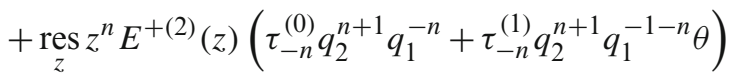

$$
\begin{aligned}
& \otimes z^{m} E^{-(2)}(z)\left(\tau_{-m}^{(0)} q_{2}^{m-1} q_{1}^{-m}+\tau_{-m}^{(1)} q_{2}^{m-1} q_{1}^{-m-1} \theta\right)
\end{aligned}
$$

This decomposes in a number of equations of which one is:

$$
\begin{aligned}
\operatorname{res}_{z} & \left((-z)^{n-m} E^{+(1)}(z) \tau_{n}^{(a)} \otimes E^{-(1)}(z) \tau_{m}^{(a)}\right. \\
& \left.+z^{m-n-2} E^{+(2)}(z) \tau_{n+1}^{(a)} \otimes E^{-(2)}(z) \tau_{m-1}^{(a)}\right)=0
\end{aligned}
$$

for $a=0,1$ which is a version of the two-component KP equation [10,14], see also [32] and

$$
\begin{aligned}
\operatorname{res}_{z}\left(z^{1-2 \delta_{a 1}}(-z)^{n-m} E^{+(1)}(z) \tau_{n}^{(a)} \otimes E^{-(1)}(z) \tau_{m}^{(1-a)}\right. \\
\left.+z^{m-n-2} E^{+(2)}(z) \tau_{n+1}^{(a)} \otimes E^{-(2)}(z) \tau_{m-1}^{(1-a)}\right) \\
=\delta_{a 1} \tau_{n}^{(0)} \otimes \tau_{m}^{(1)}
\end{aligned}
$$

which is a version of the two-component first modified KP equation [10,15].

We have

$$
\begin{aligned}
& \tau_{n}^{(0)}=\left\langle n,-n, 0\left|e^{\sum_{n=1}^{\infty}\left(t_{n}^{(1)} \alpha_{n}^{(1)}+t_{n}^{(2)} \alpha_{n}^{(2)}\right)} g\right| 0,0,0\right\rangle \quad \text { and } \\
& \tau_{n}^{(1)}=\left\langle n-1,-n, 1\left|e^{\sum_{n=1}^{\infty}\left(t_{n}^{(1)} \alpha_{n}^{(1)}+t_{n}^{(2)} \alpha_{n}^{(2)}\right)} g\right| 0,0,0\right\rangle
\end{aligned}
$$

Note, using (58), we can write $g=g^{(0,0)}+g^{(1)} \psi+g^{(-1)} \psi^{\dagger}+g^{(1,-1)} \psi \psi^{\dagger}$. Then, (74) and (75) can be written as follows:

$$
\begin{aligned}
& \tau_{n}^{(0)}=\left\langle n,-n, 0\left|e^{\sum_{n=1}^{\infty}\left(t_{n}^{(1)} \alpha_{n}^{(1)}+t_{n}^{(2)} \alpha_{n}^{(2)}\right)} g^{(0,0)}\right| 0,0,0\right\rangle \quad \text { and } \\
& \tau_{n}^{(1)}=\left\langle n-1,-n, 1\left|e^{\sum_{n=1}^{\infty}\left(t_{n}^{(1)} \alpha_{n}^{(1)}+t_{n}^{(2)} \alpha_{n}^{(2)}\right)} g^{(1)}\right| 0,0,0\right\rangle
\end{aligned}
$$




\section{B-Case in this New Realization}

Recall the involution $\omega$ (see (11)) on the Clifford algebra. Using the relabeling (48)-(52), this induces

$$
\begin{aligned}
\omega(\psi) & =\psi^{\dagger}, \quad \omega\left(\psi^{\dagger}\right)=\psi \\
\omega\left(\psi_{n}^{(1)}\right) & =\psi_{-1-n}^{\dagger(2)}, \quad \omega\left(\psi_{n}^{(2)}\right)=\psi_{-1-n}^{\dagger(1)}, \\
\omega\left(\psi_{n}^{\dagger(1)}\right)=\psi_{-1-n}^{(2)}, & \omega\left(\psi_{n}^{\dagger(2)}\right)=\psi_{-1-n}^{(1)} .
\end{aligned}
$$

It is straightforward to check that

$$
\omega\left(\alpha_{n}^{(1)}\right)=-\alpha_{n}^{(2)}, \quad \omega\left(\alpha_{n}^{(2)}\right)=-\alpha_{n}^{(1)} .
$$

Now define a new basis in our Clifford algebra consisting of elements that are fixed by $\omega$ and of elements $x$ with $\omega(x)=-x$

$$
\begin{aligned}
\varphi & =\frac{\psi+\psi^{\dagger}}{\sqrt{2}}, \quad \hat{\varphi}=i \frac{\psi-\psi^{\dagger}}{\sqrt{2}} \\
\psi_{j} & =\frac{\psi_{j}^{(1)}+\psi_{-1-j}^{\dagger(2)}}{\sqrt{2}}, \quad \hat{\psi}_{j}=i \frac{\psi_{j}^{(1)}-\psi_{-1-j}^{\dagger(2)}}{\sqrt{2}} \\
\psi_{j}^{\dagger} & =\frac{\psi_{j}^{\dagger(1)}+\psi_{-1-j}^{(2)}}{\sqrt{2}}, \quad \hat{\psi}_{j}^{\dagger}=-i \frac{\psi_{j}^{\dagger(1)}-\psi_{-1-j}^{(2)}}{\sqrt{2}}
\end{aligned}
$$

Then,

$$
\begin{aligned}
& {\left[\psi_{n}, \psi_{m}^{\dagger}\right]_{+}=\delta_{n, m}, \quad\left[\hat{\psi}_{n}, \hat{\psi}_{m}^{\dagger}\right]_{+}=\delta_{n, m}} \\
& \varphi^{2}=\hat{\varphi}^{2}=\frac{1}{2}
\end{aligned}
$$

all other elements anticommute.

Also,

$$
\begin{aligned}
& \psi_{j}|0\rangle=\hat{\psi}_{j}|0\rangle=\psi_{-1-j}^{\dagger}|0\rangle=\hat{\psi}_{-1-j}^{\dagger}|0\rangle=0, \quad j<0, \\
& \langle 0| \psi_{j}^{\dagger}=\langle 0| \hat{\psi}_{j}^{\dagger}=\langle 0| \psi_{-1-j}=\langle 0| \hat{\psi}_{-1-j}=0, \quad j<0, \\
& (\varphi+i \hat{\varphi})|0\rangle=0=\langle 0|(\varphi-i \hat{\varphi})
\end{aligned}
$$

Spin module for $\boldsymbol{b}_{\infty}$. Here, we consider the elements $\psi_{j}, \psi_{j}^{\dagger}, \varphi$, which are the elements invariant under $\omega$. Recall

$$
\left[\psi_{j}, \psi_{n}^{\dagger}\right]_{+}=\delta_{j, n}, \quad \varphi^{2}=\frac{1}{2}
$$

all other elements anticommute and

$$
\psi_{j}|0\rangle=\psi_{1-j}^{\dagger}|0\rangle=0=\langle 0| \psi_{j}^{\dagger}=\langle 0| \psi_{1-j}, \quad j<0
$$


The $b_{\infty}$ spin module splits into two parts, $V_{\overline{0}} \oplus V_{\overline{1}}$, where $V_{\overline{0}}$ has the highest weight vector $|\overline{0}\rangle=|0\rangle$, and $V_{\overline{1}}$ has the highest weight vector $|\overline{1}\rangle=\sqrt{2} \varphi|0\rangle$. Both $V_{\overline{0}}$ and $V_{\overline{1}}$ are irreducible highest weight modules for $b_{\infty}$, which is formed by the quadratic elements of the Clifford algebra together with 1 .

From now, we shall focus on $V_{\overline{0}}$.

As before one can define an oscillator algebra $B$

$$
\beta_{k}=\sum_{l \in \mathbb{Z}}: \psi_{l} \psi_{l+k}^{\dagger}:
$$

In $V_{\overline{0}}$, one has the following highest weight vectors if one restricts to the $B$

$$
\begin{aligned}
& |n\rangle=\left\{\begin{array}{lll}
\Psi_{2 l}|0\rangle & \text { if } & n=2 l \\
\sqrt{2} \Psi_{2 l-1} \varphi|0\rangle & \text { if } & n=2 l-1
\end{array}, \quad\langle n|=\left\{\begin{array}{lll}
\langle 0| \Psi_{2 l}^{\dagger} & \text { if } & n=2 l \\
\langle 0| \varphi \Psi_{2 l-1}^{\dagger} \sqrt{2} & \text { if } & n=2 l-1
\end{array}\right.\right. \\
& \beta_{k}|n\rangle=0=\langle 0| \beta_{-k}, \quad k>0, \quad n \in \mathbb{Z}
\end{aligned}
$$

where similar to (61)

$$
\Psi_{n}=\left\{\begin{array}{lll}
\psi_{n-1} \ldots \psi_{0} & \text { if } & n>0 \\
1 & \text { if } & n=0 \\
\psi_{n}^{\dagger} \ldots \psi_{-1}^{\dagger} & \text { if } & n<0
\end{array} \quad \quad \Psi_{n}^{\dagger}=\left\{\begin{array}{lll}
\psi_{0}^{\dagger} \ldots \psi_{n-1}^{\dagger} & \text { if } & n>0 \\
1 & \text { if } & n=0 \\
\psi_{-1} \ldots \psi_{n} & \text { if } & n<0
\end{array}\right.\right.
$$

Let $h$ be a group element of $B_{\infty}$. Then we can consider $h|\overline{0}\rangle$, clearly such an element decomposes

$$
h|0\rangle=\sum_{n \in \mathbb{Z}} h_{n}|n\rangle
$$

As for $G L_{\infty}$ there is a boson-fermion correspondence given by

$$
\begin{aligned}
\sigma_{B}(\psi(z)) & =p z^{p \frac{\partial}{\partial p}} B^{+}(z), \quad \sigma_{B}\left(\psi^{\dagger}(z)\right)=p^{-1} z^{-p \frac{\partial}{\partial p}} B^{-}(z) \\
B^{ \pm}(z) & =e^{ \pm \sum_{n>0} s_{n} z^{n}} e^{\mp \sum_{n>0} \frac{1}{n} \frac{\partial}{\partial s_{n}} z^{-n}}
\end{aligned}
$$

for the fermionic fields (cf. (66))

$$
\psi(z)=\sum_{i \in \mathbb{Z}} \psi_{i} z^{i}, \quad \psi^{\dagger}(z)=\sum_{i \in \mathbb{Z}} \psi_{i}^{\dagger} z^{-i-1},
$$

and (67) induces

$$
\sigma_{B}(\varphi)=\frac{\theta+\frac{\partial}{\partial \theta}}{\sqrt{2}}
$$

Then, $\sigma_{B}(h|0\rangle)=\tau^{B}$. And using (96) one finds

$$
\tau^{B}=\sum_{l \in \mathbb{Z}}\left(\tau_{2 l}^{B} p^{2 l}+\tau_{2 l-1}^{B} p^{2 l-1} \theta\right)
$$


Note that $p \theta=-\theta p$.

An element $h \in B_{\infty}$ is an even element in the Clifford algebra.

We have

$$
\left[h \otimes h, S_{B}\right]_{-}=0, \quad S_{B}=\varphi \otimes \varphi+\sum_{i \in \mathbb{Z}}\left(\psi_{i} \otimes \psi_{i}^{\dagger}+\psi_{i}^{\dagger} \otimes \psi_{i}\right)
$$

Since

$$
S_{B}|0\rangle \otimes|0\rangle=\varphi|0\rangle \otimes \varphi|0\rangle
$$

One obtains

$$
S_{B}(h|0\rangle \otimes h|0\rangle)=h \varphi|0\rangle \otimes h \varphi|0\rangle
$$

Note that $h=a+\sqrt{2} b \varphi$ where $a$ is an even element expressed in $\psi_{i}$ and $\psi_{i}^{\dagger}$, and $b$ is an odd element expressed in $\psi_{i}$ and $\psi_{i}^{\dagger}$. Then,

$$
S_{B}(a+\sqrt{2} b \varphi)|0\rangle \otimes(a+\sqrt{2} b \varphi)|0\rangle=\left(a \varphi+\frac{1}{2} \sqrt{2} b\right)|0\rangle \otimes\left(a \varphi+\frac{1}{2} \sqrt{2} b\right)|0\rangle
$$

or

$$
\begin{aligned}
& \left(a \varphi-\frac{1}{2} \sqrt{2} b\right)|0\rangle \otimes\left(a \varphi-\frac{1}{2} \sqrt{2} b\right)|0\rangle \\
& +\sum_{i \in \mathbb{Z}} \psi_{i}(a+\sqrt{2} b \varphi)|0\rangle \otimes \psi_{i}^{\dagger}(a+\sqrt{2} b \varphi)|0\rangle \\
& +\sum_{i \in \mathbb{Z}} \psi_{i}^{\dagger}(a+\sqrt{2} b \varphi)|0\rangle \otimes \psi_{i}(a+\sqrt{2} b \varphi)|0\rangle \\
& =\left(a \varphi+\frac{1}{2} \sqrt{2} b\right)|0\rangle \otimes\left(a \varphi+\frac{1}{2} \sqrt{2} b\right)|0\rangle
\end{aligned}
$$

Hence,

$$
\begin{aligned}
& \sum_{i \in \mathbb{Z}}\left[\psi_{i}(a+\sqrt{2} b \varphi)|0\rangle \otimes \psi_{i}^{\dagger}(a+\sqrt{2} b \varphi)|0\rangle+\psi_{i}^{\dagger}(a+\sqrt{2} b \varphi)|0\rangle \otimes \psi_{i}(a+\sqrt{2} b \varphi)|0\rangle\right] \\
& \quad=\sqrt{2} a \varphi|0\rangle \otimes b|0\rangle+b|0\rangle \otimes \sqrt{2} a \varphi|0\rangle
\end{aligned}
$$

Now clearly:

$$
\begin{aligned}
\sigma(a|0\rangle) & =\sum_{l \in \mathbb{Z}} \tau_{2 l}^{B} p^{2 l}, \quad \sigma(\sqrt{2} b \varphi|0\rangle)=\sum_{l \in \mathbb{Z}} \tau_{2 l-1}^{B} p^{2 l-1} \theta \\
\sigma(\sqrt{2} a \varphi|0\rangle) & =\sum_{l \in \mathbb{Z}} \tau_{2 l}^{B} p^{2 l} \theta, \quad \sigma(b|0\rangle)=\sum_{l \in \mathbb{Z}} \tau_{2 l-1}^{B} p^{2 l-1}
\end{aligned}
$$

Also,

$$
\tau_{l}^{B}(s)=\left\langle l\left|e^{\sum_{l} s_{l} \beta_{l}} h\right| 0\right\rangle
$$


Equation (105) turns into the large BKP hierarchy or Pfaff lattice:

$$
\begin{aligned}
& \underset{z}{\operatorname{res}}\left(z^{n-m-2} B^{+}(z) \tau_{n-1}^{B} \otimes B^{-}(z) \tau_{m+1}^{B}\right. \\
& \left.\quad+\frac{(-1)^{n+m}}{2 z} \tau_{n}^{B} \otimes \tau_{m}^{B}+z^{m-n-2} B^{-}(z) \tau_{n+1}^{B} \otimes B^{+}(z) \tau_{m-1}^{B}\right) \\
& \quad=\frac{1}{2} \tau_{n}^{B} \otimes \tau_{m}^{B}
\end{aligned}
$$

\section{A Relation Between the Tau Functions}

In the same way as the small BKP is related to the KP hierarchy, the large BKP (or Pfaff lattice) is related to the two-component KP (or Toda Lattice hierarchy). In fact, one can use the same involution ^ of (28) on the Clifford algebra, it induces

$$
\begin{aligned}
& { }^{\wedge}(\varphi)=\hat{\varphi}, \quad{ }^{\prime}(\hat{\varphi})=\varphi \\
& \hat{\imath}\left(\psi_{i}\right)=\hat{\psi}_{i}, \quad{ }^{\wedge}\left(\hat{\psi}_{i}\right)=\psi_{i}, \quad{ }^{\wedge}\left(\psi_{i}^{\dagger}\right)=\hat{\psi}_{i}^{\dagger}, \quad{ }^{\prime}\left(\hat{\psi}_{i}^{\dagger}\right)=\hat{\psi}_{i}^{\dagger}
\end{aligned}
$$

Then,

$$
\hat{\imath}^{\wedge}\left(\beta_{l}\right)=\hat{\beta}_{l}=\sum_{j \in \mathbb{Z}}: \hat{\psi}_{j} \hat{\psi}_{j+l}^{\dagger}:
$$

We want to consider

$$
e^{\sum_{l} s_{l}\left(\beta_{l}+\hat{+}\left(\beta_{l}\right)\right)} h \cdot \hat{*}(h)|0\rangle=e^{\sum_{l} s_{l} \beta_{l}} h \cdot e^{\sum_{l} s_{l} \hat{\beta}_{l}} \hat{h}|0\rangle
$$

Now,

$$
\begin{aligned}
\psi_{j} \psi_{k}^{\dagger}+\hat{\psi}_{j} \hat{\psi}_{k}^{\dagger}= & \frac{1}{2}\left(\psi_{j}^{(1)}+\psi_{-j-1}^{\dagger(2)}\right)\left(\psi_{k}^{\dagger(1)}+\psi_{-k-1}^{(2)}\right) \\
& +\frac{1}{2}\left(\psi_{j}^{(1)}-\psi_{-j-1}^{\dagger(2)}\right)\left(\psi_{k}^{\dagger(1)}-\psi_{-k-1}^{(2)}\right) \\
= & \psi_{j}^{(1)} \psi_{k}^{\dagger(1)}+\psi_{-j-1}^{(2)} \psi_{-k-1}^{\dagger(2)}
\end{aligned}
$$

Thus,

$$
\begin{aligned}
\beta_{l}+\hat{\beta}_{l} & =\sum_{j}\left(: \psi_{j} \psi_{j+l}^{\dagger}:+: \hat{\psi}_{j} \hat{\psi}_{j+l}^{\dagger}:\right)=\sum_{j}\left(: \psi_{j}^{(1)} \psi_{j+l}^{\dagger(1)}:+: \psi_{-j-1}^{\dagger(2)} \psi_{-j-l-1}^{(2)}:\right) \\
& =\alpha_{l}^{(1)}-\alpha_{j}^{(2)}
\end{aligned}
$$

and hence $e^{\sum s_{l}\left(\beta_{l}+\hat{\beta}_{l}\right)}=e^{\sum_{l} s_{l} \alpha_{l}^{(1)}} e^{-\sum_{l} s_{l} \alpha_{l}^{(2)}}$

First, note that

$$
\psi=\frac{\varphi-i \hat{\varphi}}{\sqrt{2}}, \quad \psi^{\dagger}=\frac{\varphi+i \hat{\varphi}}{\sqrt{2}}
$$




$$
\begin{aligned}
& \psi_{j}^{(1)}=\frac{\psi_{j}-i \hat{\psi}_{j}}{\sqrt{2}}, \quad \psi_{j}^{\dagger(1)}=\frac{\psi_{j}^{\dagger}+i \hat{\psi}_{j}^{\dagger}}{\sqrt{2}} \\
& \psi_{j}^{(2)}=\frac{\psi_{-j-1}^{\dagger}-i \hat{\psi}_{-j-1}^{\dagger}}{\sqrt{2}}, \quad \psi_{j}^{\dagger(2)}=\frac{\psi_{-j-1}+i \hat{\psi}_{-j-1}}{\sqrt{2}}
\end{aligned}
$$

Thus,

$$
\begin{gathered}
\psi_{j}^{(1)} \psi_{-j-1}^{\dagger(2)}=\frac{1}{2}\left(\psi_{j}-i \hat{\psi}_{j}\right)\left(\psi_{j}+i \hat{\psi}_{j}\right)=i \psi_{j} \hat{\psi}_{j} \\
\psi_{j}^{\dagger(1)} \psi_{-j-1}^{(2)}=\frac{1}{2}\left(\psi_{j}^{\dagger}+i \hat{\psi}_{j}^{\dagger}\right)\left(\psi_{j}^{\dagger}-i \hat{\psi}_{j}^{\dagger}\right)=-i \psi_{j}^{\dagger} \hat{\psi}_{j}^{\dagger}
\end{gathered}
$$

The following Lemma will be useful:

\section{LEMMA 1.}

$$
\begin{aligned}
\langle-2 n, 2 n, 0| & =(-1)^{n}\langle 0| \Psi_{-2 n}^{\dagger} \hat{\Psi}_{-2 n}^{\dagger} \\
\langle 1-2 n, 2 n-1,0| & =(-1)^{n+1}\langle 0|\left(\sqrt{2} \varphi \Psi_{1-2 n}^{\dagger}\right)\left(\sqrt{2} \hat{\varphi} \hat{\Psi}_{1-2 n}^{\dagger}\right)
\end{aligned}
$$

Proof. Let $n>0$. Then,

$$
\begin{aligned}
\langle-2 n, 2 n, 0| & =\langle 0| \Psi_{-2 n}^{\dagger(1)} \Psi_{2 n}^{\dagger(2)}=\langle 0| \psi_{-1}^{(1)} \psi_{-2}^{(1)} \ldots \psi_{-2 n}^{(1)} \psi_{0}^{\dagger(2)} \psi_{1}^{\dagger(2)} \ldots \psi_{2 n-1}^{\dagger(2)} \\
& =(-1)^{n}\langle 0| \psi_{-1} \ldots \psi_{-2 n} \hat{\psi}_{-1} \ldots \hat{\psi}_{-2 n}=(-1)^{n}\langle 0| \Psi_{-2 n}^{\dagger} \hat{\Psi}_{-2 n}^{\dagger}
\end{aligned}
$$

If $n<0$, then we have

$$
\begin{aligned}
\langle-2 n, 2 n, 0| & =\langle 0| \Psi_{-2 n}^{\dagger(1)} \Psi_{2 n}^{\dagger(2)}=\langle 0| \psi_{0}^{\dagger(1)} \psi_{1}^{\dagger(1)} \ldots \psi_{-2 n-1}^{\dagger(1)} \psi_{-1}^{(2)} \psi_{-2}^{(2)} \ldots \psi_{2 n}^{(2)} \\
& =(-1)^{n}\langle 0| \psi_{0}^{\dagger} \psi_{1}^{\dagger} \ldots \psi_{-2 n-1}^{\dagger} \hat{\psi}_{0}^{\dagger} \ldots \hat{\psi}_{-2 n-1}^{\dagger}=(-1)^{n}\langle 0| \Psi_{-2 n}^{\dagger} \hat{\Psi}_{-2 n}^{\dagger}
\end{aligned}
$$

Next, we consider the case

$$
\langle 1-2 n, 2 n-1,0|=\langle 0| \Psi_{-2 n+1}^{\dagger(1)} \Psi_{2 n-1}^{\dagger(2)}
$$

If $n>0$, then:

$$
\begin{aligned}
\langle 1-2 n, 2 n-1,0| & =\langle 0| \psi_{-1}^{(1)} \ldots \psi_{-2 n+1}^{(1)} \psi_{0}^{\dagger(2)} \ldots \psi_{2 n-2}^{\dagger(2)} \\
& =(i)^{2 n-1}\langle 0| \psi_{-1} \ldots \psi_{-2 n+1} \hat{\psi}_{-1} \ldots \hat{\psi}_{-2 n+1} \\
& =(i)^{2 n-1}\langle 0| \Psi_{-2 n+1}^{\dagger} \hat{\Psi}_{-2 n+1}^{\dagger}
\end{aligned}
$$

Now, use that

$$
\langle 0| \varphi \hat{\varphi}=\frac{i}{2}\langle 0|\left(\psi+\psi^{\dagger}\right)\left(\psi+\psi^{\dagger}\right)=-\frac{i}{2}\langle 0| \psi^{\dagger} \psi=-\frac{i}{2}\langle 0|
$$


Thus,

$$
\begin{aligned}
\langle 1 & -2 n, 2 n-1,0 \mid=2(i)^{2 n}\langle 0| \varphi \hat{\varphi} \Psi_{-2 n+1}^{\dagger} \hat{\Psi}_{-2 n+1}^{\dagger} \\
= & (-1)^{n+1}\langle 0|\left(\sqrt{2} \varphi \Psi_{1-2 n}^{\dagger}\right)\left(\sqrt{2} \hat{\varphi} \hat{\Psi}_{1-2 n}^{\dagger}\right)
\end{aligned}
$$

If $n<0$, then

$$
\begin{aligned}
\langle 1-2 n, 2 n-1,0| & =\langle 0| \psi_{0}^{\dagger(1)} \ldots \psi_{2 n}^{\dagger(1)} \psi_{-1}^{(2)} \ldots \psi_{2 n-1}^{(2)} \\
& =(-i)^{1-2 n}\langle 0| \psi_{0}^{\dagger} \ldots \psi_{-2 n}^{\dagger} \hat{\psi}_{0}^{\dagger} \ldots \hat{\psi}_{-2 n}^{\dagger} \\
& =(-i)^{1-2 n}\langle 0| \Psi_{1-2 n}^{\dagger} \hat{\Psi}_{1-2 n}^{\dagger} \\
& =(-1)^{n+1}\langle 0|\left(\sqrt{2} \varphi \Psi_{1-2 n}^{\dagger}\right)\left(\sqrt{2} \hat{\varphi} \hat{\Psi}_{1-2 n}^{\dagger}\right)
\end{aligned}
$$

Which finishes the proof of the lemma

Thus,

$$
\begin{aligned}
\tau_{-2 n}^{(0)}(s,-s) & =(-1)^{n}\left\langle 0\left|\Psi_{-2 n}^{\dagger} \hat{\Psi}_{-2 n}^{\dagger} e^{\sum_{k} s_{k} \beta_{k}} e^{\sum_{k} s_{k} \hat{\beta}_{k}} h \hat{h}\right| 0\right\rangle \\
& =(-1)^{n}\left\langle-2 n\left|e^{\sum_{k} s_{k} \beta_{k}} h\right| 0\right\rangle\left\langle-2 n\left|e^{\sum_{k} s_{k} \hat{\beta}_{k}} \hat{h}\right| 0\right\rangle=(-1)^{n} \tau_{2 n}^{B}(s) \tau_{2 n}^{B}(s)
\end{aligned}
$$

and

$$
\begin{aligned}
\tau_{1-2 n}^{(0)}(s,-s) & =\left\langle 1-2 n, 2 n-1,0\left|e^{\sum_{k} s_{k} \beta_{k}} e^{\sum_{k} s_{k} \hat{\beta}_{k}} h \hat{h}\right| 0\right\rangle \\
& =(-1)^{n+1}\left\langle 1-2 n\left|e^{\sum_{k} s_{k} \beta_{k}} h\right| 0\right\rangle\left\langle\widehat{1-2 n}\left|e^{\sum_{k} s_{k} \hat{\beta}_{k}} \hat{h}\right| 0\right\rangle \\
& =(-1)^{n+1} \tau_{1-2 n}^{B}(s) \tau_{1-2 n}^{B}(s)
\end{aligned}
$$

Thus,

PROPOSITION 1. A BKP tau function satisfies

$$
\left(\tau_{n}^{B}(s)\right)^{2}=(-)^{\frac{n(n+1)}{2}} \tau_{n}^{(0)}(s,-s)
$$

Remark 2. If $g_{i}=h_{i} \hat{h}_{i}, i=1, \ldots, k$, then $g=g_{1} \ldots g_{k}=h \hat{h}$ where $h=h_{1} \ldots h_{k}$ and $\hat{h}=\hat{h}_{1} \ldots \hat{h}_{k}$.

\section{A Relation Between the Wave Functions}

We introduce the two-component $\mathrm{KP}$ wave function $W_{n}^{ \pm(a)}(t, z)$ by

$$
W_{n}^{ \pm(a)}(t, z)=\frac{1}{\tau_{n}^{(a)}(t)}\left(\begin{array}{ll}
(-z)^{ \pm n} E^{ \pm(1)}(z) \tau_{n}^{(a)}(t) & z^{\mp n-1} E^{ \pm(2)}(z) \tau_{n \pm 1}^{(a)}(t) \\
(-z)^{ \pm n-1} E^{ \pm(1)}(z) \tau_{n \mp 1}^{(a)}(t) & z^{\mp n} E^{ \pm(2)}(z) \tau_{n}^{(a)}(t)
\end{array}\right)
$$

then (72) turns into

$$
\operatorname{res}_{z} W_{n}^{+(a)}(s, z) W_{m}^{-(a)}(t, z)^{T}=0
$$


and (73) turns into

$$
\begin{gathered}
\underset{z}{\operatorname{res}} W_{n}^{+(a)}(s, z)\left(\begin{array}{cc}
z^{1-2 \delta_{a 1}} & 0 \\
0 & 1
\end{array}\right) W_{m}^{-(1-a)}(t, z)^{T} \\
=\frac{\delta_{a 1}}{\tau_{n}^{(1)}(s) \tau_{m}^{(0)}(t)}\left(\begin{array}{c}
\tau_{n}^{(0)}(s) \\
\tau_{n-1}^{(0)}(s)
\end{array}\right)\left(\tau_{m}^{(1)}(t) \tau_{m+1}^{(1)}(t)\right)
\end{gathered}
$$

We introduce next the BKP wave function $V_{n}^{ \pm}(z)$. To do that, we first observe that (109) can be rewritten in the matrix form

$$
\underset{z}{\operatorname{res}} R_{n}^{+}(z) S(z) R_{m}^{-}(z)^{T}=\underset{z}{\operatorname{res}} T_{n}^{+} S(z) T_{m}^{-T}
$$

where

$$
\begin{gathered}
R_{n}^{ \pm}(z)=\left(\begin{array}{lll}
z^{ \pm n} B^{ \pm}(z) \tau_{n}^{B} & (-1)^{n+1} \tau_{n \pm 1}^{B} & z^{\mp n-2} B^{\mp}(z) \tau_{n \pm 2}^{B} \\
z^{ \pm n-1} B^{ \pm}(z) \tau_{n \mp 1}^{B} & (-1)^{n} \tau_{n}^{B} & z^{\mp n-1} B^{\mp}(z) \tau_{n \pm 1}^{B} \\
z^{ \pm n-2} B^{ \pm}(z) \tau_{n \mp 2}^{B} & (-1)^{n-1} \tau_{n \mp 1}^{B} & z^{\mp n} B^{\mp}(z) \tau_{n}^{B}
\end{array}\right) \\
T_{n}^{ \pm}=\left(\begin{array}{lll}
\tau_{n}^{B} & \tau_{n \pm 1}^{B} & 0 \\
0 & \tau_{n}^{B} & 0 \\
0 & \tau_{n \mp 1}^{B} & \tau_{n}^{B}
\end{array}\right)
\end{gathered}
$$

and

$$
S(z)=\operatorname{diag}\left(1, \frac{1}{2 z}, 1\right)
$$

Let

$$
U_{n}^{ \pm}=\left(\begin{array}{clc}
\frac{1}{\tau_{n}^{B}} & \mp \frac{\tau_{n \pm 1}^{B}}{\left(\tau_{n}^{B}\right)^{2}} & 0 \\
0 & \frac{1}{\tau_{n}^{B}} & 0 \\
0 & \mp \frac{\tau_{n \mp 1}^{B}}{\left(\tau_{n}^{B}\right)^{2}} & \frac{1}{\tau_{n}^{B}}
\end{array}\right)
$$

then $U_{n}^{+}=\left(T_{n}^{+}\right)^{-1}$. Now introduce the BKP wave function $V_{n}^{ \pm}(z)=U_{n}^{ \pm} R_{n}^{ \pm}(z)$ then (128) turns into

$$
\underset{z}{\operatorname{res}} V_{n}^{+}(z) S(z) V_{m}^{-}(z)^{T}=\left(\begin{array}{ccc}
0 & 0 & 0 \\
\frac{\tau_{m-1}^{B}}{\tau_{m}^{B}} & \frac{1}{2} & \frac{\tau_{m+1}^{B}}{\tau_{m}^{B}} \\
0 & 0 & 0
\end{array}\right)
$$

We will now show that two-component $\mathrm{KP}$ wave functions $W_{n}^{ \pm(0)}\left(t^{(1)}, t^{(2)}, z\right)$ evaluated at $t_{j}^{(1)}=s_{j}=-t^{(2)}$ for group elements, $g=h \hat{h}$ may be expressed in terms of the BKP wave functions $V_{n}^{ \pm}(z)$.

PROPOSITION 2. A BKP wave function is related to a two-component KP wave function, via

$$
W_{n}^{ \pm(0)}(s,-s, z)=\left(\begin{array}{lll}
(-1)^{n} & 0 & 0 \\
0 & 0 & \mp 1
\end{array}\right) V_{n}^{ \pm}(s, z)\left(\begin{array}{ll}
1 & 0 \\
0 & 0 \\
0 & \mp 1
\end{array}\right)
$$


To prove this, we calculate

$$
\begin{aligned}
w_{n}^{(1)}\left(t^{(1)}, t^{(2)}, z\right) & =\left\langle n+1,-n, 0\left|e^{\sum_{j>0}\left(t_{j}^{(1)} \alpha_{j}+t_{j}^{(2)} \hat{\alpha}_{j}\right)} \psi^{(1)}(z) g\right| 0\right\rangle \\
& =(-z)^{n} e^{\sum_{j>0} t_{j}^{(1)} z^{j}}\left\langle n,-n, 0\left|e^{\sum_{j>0}\left(\left(t_{j}^{(1)}-\frac{z^{-j}}{j}\right) \alpha_{j}+t_{j}^{(2)} \hat{\alpha}_{j}\right)} g\right| 0\right\rangle \\
& =(-z)^{n} e^{\sum_{j>0} t_{j}^{(1)} z^{j}} \tau_{n}^{(0)}\left(t^{(1)}-\left[z^{-1}\right], t^{(2)}\right)
\end{aligned}
$$

Now, set $g=h \hat{h}$ and $t_{j}^{(1)}=s_{j}=-t^{(2)}$. Then

$$
\begin{aligned}
& w_{n}^{(1)}(s,-s, z)=(-z)^{n} e^{\sum_{j>0} s_{j} z^{j}} \tau_{n}^{(0)}\left(s-\left[z^{-1}\right],-s\right) \\
&=\left\langle n+1,-n, 0\left|e^{\sum_{j>0} s_{j}\left(\beta_{j}+\hat{\beta}_{j}\right)} \psi^{(1)}(z) h \hat{h}\right| 0\right\rangle \\
&=(-i)^{n+1}\left\langle 0\left|\Psi_{n+1}^{\dagger} \hat{\Psi}_{n+1}^{\dagger} \psi_{-n-1}^{\dagger}(2) e^{\sum_{j>0} s_{j}\left(\beta_{j}+\hat{\beta}_{j}\right)} \psi^{(1)} h \hat{h}\right| 0\right\rangle \\
&= \frac{(-i)^{n+1}}{2}\left\langle 0\left|\Psi_{n+1}^{\dagger} \hat{\Psi}_{n+1}^{\dagger}\left(\psi_{n}+i \hat{\psi}_{n}\right) e^{\sum_{j>0} s_{j}\left(\beta_{j}+\hat{\beta}_{j}\right)}(\psi(z)-i \hat{\psi}(z)) h \hat{h}\right| 0\right\rangle \\
&=\left(\frac{i^{n+1}}{2}\left\langle 0\left|\Psi_{n}^{\dagger} \hat{\Psi}_{n+1}^{\dagger}+\frac{(-i)^{n}}{2}\langle 0| \Psi_{n}^{\dagger} \hat{\Psi}_{n+1}^{\dagger}\right) e^{\sum_{j>0} s_{j}\left(\beta_{j}+\hat{\beta}_{j}\right)}(\psi(z)-i \hat{\psi}(z)) h \hat{h} \mid 0\right\rangle\right. \\
&= \frac{(-i)^{n+1}}{2} z^{n-1} e^{\sum_{j>0} s_{j} z^{j}}\left\langle 0\left|\Psi_{n-1}^{\dagger} \hat{\Psi}_{n+1}^{\dagger} e^{\left.\sum_{j>0}\left(s_{j}-\frac{z^{-j}}{j}\right) \beta_{j}+s_{j} \hat{\beta}_{j}\right)} h \hat{h}\right| 0\right\rangle \\
&+\frac{i^{n}}{2} z^{n} e^{\sum_{j>0} s_{j} z^{j}}\left\langle 0\left|\Psi_{n}^{\dagger} \hat{\Psi}_{n}^{\dagger} e^{\left.\sum_{j>0}\left(s_{j}-\frac{z^{-j}}{j}\right) \beta_{j}+s_{j} \hat{\beta}_{j}\right)} h \hat{h}\right| 0\right\rangle \\
&+\frac{i^{n}}{2} z^{n} e^{\sum_{j>0} s_{j} z^{j}}\left\langle 0\left|\Psi_{n}^{\dagger} \hat{\Psi}_{n}^{\dagger} e^{\left.\sum_{j>0} s_{j} \beta_{j}+\left(s_{j}-\frac{z^{-j}}{j}\right) \hat{\beta}_{j}\right)} h \hat{h}\right| 0\right\rangle \\
&+\frac{(-i)^{n+1}}{2} z^{n-1} e^{\sum_{j>0} s_{j} z^{j}}\left\langle 0\left|\Psi_{n+1}^{\dagger} \hat{\Psi}_{n-1}^{\dagger} e^{\left.\sum_{j>0} s_{j} \beta_{j}+\left(s_{j}-\frac{z^{-j}}{j}\right) \hat{\beta}_{j}\right)} h \hat{h}\right| 0\right\rangle \\
&=\{(-1)^{\frac{n}{2}} z^{n} e^{\sum_{j>0} s_{j} z^{j}}\left(\tau_{n}^{B}(s) \tau_{n}^{B}\left(s-\left[z^{-1}\right]\right)-z^{-1} \tau_{n+1}^{B}(s) \tau_{n-1}^{B}\left(s-\left[z^{-1}\right]\right)\right), \quad \text { if } \quad n \text { even } \\
&(-1)^{\frac{n-1}{2}} z^{n} e^{\sum_{j>0} s_{j} z^{j}}\left(\tau_{n}^{B}(s) \tau_{n}^{B}\left(s-\left[z^{-1}\right]\right)-z^{-1} \tau_{n+1}^{B}(s) \tau_{n-1}^{B}\left(s-\left[z^{-1}\right]\right)\right), \quad \text { if } \quad n \text { odd }
\end{aligned}
$$

Thus, we obtain

$$
\begin{aligned}
w_{n}^{(1)}(s,-s, z)= & (-1)^{\frac{n(n-1)}{2}} z^{n} e^{\sum_{j>0} s_{j} z^{j}} \\
& \times\left(\tau_{n}^{B}(s) \tau_{n}^{B}\left(s-\left[z^{-1}\right]\right)-z^{-1} \tau_{n+1}^{B}(s) \tau_{n-1}^{B}\left(s-\left[z^{-1}\right]\right)\right)
\end{aligned}
$$

which is the first formula of

$$
\begin{aligned}
& (-1)^{\frac{n(n+1)}{2}} \tau_{n}^{(0)}\left(s-\left[z^{-1}\right],-s\right)=\tau_{n}^{B}(s) \tau_{n}^{B}\left(s-\left[z^{-1}\right]\right)-z^{-1} \tau_{n+1}^{B}(s) \tau_{n-1}^{B}\left(s-\left[z^{-1}\right]\right) \\
& (-1)^{\frac{n(n+1)}{2}} \tau_{n}^{(0)}\left(s+\left[z^{-1}\right],-s\right)=\tau_{n}^{B}(s) \tau_{n}^{B}\left(s+\left[z^{-1}\right]\right)+z^{-1} \tau_{n-1}^{B}(s) \tau_{n+1}^{B}\left(s+\left[z^{-1}\right]\right)
\end{aligned}
$$




$$
\begin{aligned}
& (-1)^{\frac{n(n+1)}{2}} \tau_{n}^{(0)}\left(s,-s-\left[z^{-1}\right]\right)=\tau_{n}^{B}(s) \tau_{n}^{B}\left(s+\left[z^{-1}\right]\right)-z^{-1} \tau_{n-1}^{B}(s) \tau_{n+1}^{B}\left(s+\left[z^{-1}\right]\right) \\
& \left.(-1)^{\frac{n(n+1)}{2}} \tau_{n}^{(0)}\left(s,-s+\left[z^{-1}\right]\right)\right)=\tau_{n}^{B}(s) \tau_{n}^{B}\left(s-\left[z^{-1}\right]\right)+z^{-1} \tau_{n+1}^{B}(s) \tau_{n-1}^{B}\left(s-\left[z^{-1}\right]\right)
\end{aligned}
$$

The other formulas can be obtained in a similar way. From these formulas and Proposition 1, one easily deduces Proposition 2.

\section{The Two-Sided BKP (2-BKP) and Two-Component Toda Lattice}

In this section, we consider a two-sided version of some of the previous constructions.

\section{2-BKP and two-component 2-KP (two-component Toda lattice) tau functions.}

Consider

$$
\begin{aligned}
& \tau_{n, m}^{(0)}\left(t^{(1)}, t^{(2)} ; \bar{t}^{(1)}, \bar{t}^{(2)} \mid g\right) \\
& \quad:=\left\langle n,-n, 0\left|e^{\sum_{i>0}\left(t_{i}^{(1)} \alpha_{i}^{(1)}+t_{i}^{(2)} \alpha_{i}^{(2)}\right)} g e^{\sum_{i>0}\left(\bar{t}_{i}^{(1)} \alpha_{-i}^{(1)}+\bar{t}_{i}^{(2)} \alpha_{-i}^{(2)}\right.}\right| m,-m, 0\right\rangle
\end{aligned}
$$

which may be considered as the two-component 2-KP tau function, or, the same, two-component Toda lattice tau function. ${ }^{1}$ The Hirota equations for the tau function (140) may also be found in the Appendix A.3

In what follows, we shall put $t_{k}^{(1)}=s_{k}=-t_{k}^{(2)}, \bar{t}_{k}^{(1)}=\bar{s}_{k}=-\bar{t}_{k}^{(2)}$, where $s$ and $\bar{s}$ are two independent sets of variables. Below $g=h \hat{h}$. These are restrictions which allow to compare two-component TL tau functions with BKP tau functions.

Consider a 2-BKP tau function

$$
\tau_{n, m}^{B}(s, \bar{s} \mid h):=\left\langle n\left|e^{\sum_{i>0} s_{i} \beta_{i}} h e^{\sum_{i>0} \bar{s}_{i} \beta_{-i}}\right| m\right\rangle
$$

which depends on two discrete parameters $n$ and $m$ and two sets of higher times $s=\left(s_{1}, s_{2}, \ldots\right)$ and $\bar{s}=\left(\bar{s}_{1}, \bar{s}_{2}, \ldots\right)$. Hirota equations of the 2-BKP. The name 2BKP (two-sided BKP) is related to the fact that 2-BKP Hirota equations (see Appendix A.4) contains the BKP Hirota equations with respect to the variables $s, m$ the same as Hirota BKP equations with respect the variables $\bar{s}, n$ (109).

Remark 3. We have the following symmetry

$$
\tau_{n, m}^{B}(s, \bar{s} \mid h)=\tau_{m, n}^{B}\left(\bar{s}, s \mid h^{\dagger}\right)
$$

Then it follows that the 2-BKP tau functions $(141) \tau_{n, m}(s, \bar{s})$ is a BKP tau function with respect to the variables $\bar{s}$. This explains the name 2-BKP tau function.

In the same way as Proposition 1 was obtained, we get

\footnotetext{
${ }^{1}$ See a piece between relations (9.6) and (9.7) in [10].
} 
PROPOSITION 3. A BKP tau function satisfies

$$
\left(\tau_{n, m}^{B}(s, \bar{s} \mid h)\right)^{2}=(-)^{\frac{n(n+1)}{2}+\frac{m(m+1)}{2}} \tau_{n, m, 0}^{(0)}(s,-s ; \bar{s},-\bar{s} \mid g)
$$

where $g=h \hat{h}$.

For proof of Proposition 3, we note that from Lemma 1 it follows that

$$
\begin{aligned}
& |-2 n, 2 n, 0\rangle=(-1)^{n} \hat{\Psi}_{-2 n} \Psi_{-2 n}|0\rangle, \\
& \quad|1-2 n, 2 n-1,0\rangle=(-1)^{n+1} \hat{\Psi}_{1-2 n} \sqrt{2} \hat{\varphi} \Psi_{1-2 n} \sqrt{2} \varphi|0\rangle
\end{aligned}
$$

which allows to repeat all the steps of the derivation of the Proposition 1.

Miwa transforms For tau functions of the two-component TL and 2-BKP, we can write (cf. (136-139)):

$$
\begin{aligned}
& (-1)^{\frac{n(n+1)}{2}}+\frac{m(m+1)}{2} \tau_{n, m, 0}^{(0)}(s-[x],-s ; \bar{s},-\bar{s}) \\
& =\tau_{n, m}^{B}(s, \bar{s}) \tau_{n, m}^{B}(s-[x], \bar{s})-x \tau_{n+1, m}^{B}(s, \bar{s}) \tau_{n-1, m}^{B}(s-[x], \bar{s}) \\
& (-1)^{\frac{n(n+1)}{2}+\frac{m(m+1)}{2}} \tau_{n, m, 0}^{(0)}(s+[x],-s ; \bar{s},-\bar{s}) \\
& \left.=\tau_{n, m}^{B}(s, \bar{s}) \tau_{n, m}^{B}(s+[x], \bar{s}]\right)+x \tau_{n-1, m}^{B}(s, \bar{s}) \tau_{n+1, m}^{B}(s+[x], \bar{s}) \\
& (-1)^{\frac{n(n+1)}{2}+\frac{m(m+1)}{2}} \tau_{n, m, 0}^{(0)}(s,-s-[x] ; \bar{s},-\bar{s}) \\
& =\tau_{n, m}^{B}(s, \bar{s}) \tau_{n, m}^{B}(s+[x], \bar{s})-x \tau_{n-1, m}^{B}(s, \bar{s}) \tau_{n+1, m}^{B}(s+[x], \bar{s}) \\
& (-1)^{\frac{n(n+1)}{2}+\frac{m(m+1)}{2}} \tau_{n, m, 0}^{(0)}(s,-s+[x] ; \bar{s},-\bar{s}) \\
& =\tau_{n, m}^{B}(s, \bar{s}) \tau_{n, m}^{B}(s-[x], \bar{s})+x \tau_{n+1, m}^{B}(s, \bar{s}) \tau_{n-1, m}^{B}(s-[x], \bar{s}) \\
& (-1)^{\frac{n(n+1)}{2}}+\frac{m(m+1)}{2} \tau_{n, m, 0}^{(0)}(s,-s ; \bar{s}-[x],-\bar{s}) \\
& =\tau_{n, m}^{B}(s, \bar{s}) \tau_{n, m}^{B}(s, \bar{s}-[x])-x \tau_{n, m+1}^{B}(s, \bar{s}) \tau_{n, m-1}^{B}(s, \bar{s}-[x]) \\
& (-1)^{\frac{n(n+1)}{2}+\frac{m(m+1)}{2}} \tau_{n, m, 0}^{(0)}(s,-s ; \bar{s}+[x],-\bar{s}) \\
& =\tau_{n, m}^{B}(s, \bar{s}) \tau_{n, m}^{B}(s, \bar{s}+[x])+x \tau_{n, m-1}^{B}(s, \bar{s}) \tau_{n, m+1}^{B}(s, \bar{s}+[x]) \\
& (-1)^{\frac{n(n+1)}{2}+\frac{m(m+1)}{2}} \tau_{n, m, 0}^{(0)}(s,-s ; \bar{s},-\bar{s}-[x]) \\
& =\tau_{n, m}^{B}(s, \bar{s}) \tau_{n, m}^{B}(s, \bar{s}+[x])-x \tau_{n, m-1}^{B}(s, \bar{s}) \tau_{n, m+1}^{B}(s, \bar{s}+[x]) \\
& \left.(-1)^{\frac{n(n+1)}{2}+\frac{m(m+1)}{2}} \tau_{n, m, 0}^{(0)}(s,-s) ; \bar{s},-\bar{s}+[x]\right) \\
& =\tau_{n, m}^{B}(s, \bar{s}) \tau_{n, m}^{B}(s, \bar{s}-[x])+x \tau_{n, m+1}^{B}(s, \bar{s}) \tau_{n, m-1}^{B}(s, \bar{s}-[x])
\end{aligned}
$$

\section{An Example: Toda Lattice (TL) and B-Type Pfaff Lattice (BPL)}

In many applications (like random matrices or random partitions), the semi-infinite Toda lattice and semi-infinite Pfaff lattice are of use. Here, we relate the semiinfinite Pfaff lattice of $B$-type to the semi-infinite Toda lattice. 
Semi-infinite Toda lattice. First, let us recall that the Hirota equation for the Toda lattice tau function [10,32] and for the two-component KP tau function [10] coincide up to a sign factor, see the relation (9.7) in [10] and the Theorem 1.12 in [32]. Here, we shall consider the semi-infinite Toda lattice which may be presented as

$$
\begin{aligned}
\tau_{n}^{\mathrm{TL}}(t, \bar{t}) & =(-)^{\frac{n(n+1)}{2}} \tau_{n}^{2 \mathrm{KP}}(t, \bar{t}) \\
\tau_{N}^{2 \mathrm{KP}}\left(t^{(1)}, t^{(2)}\right) & =\left\langle N,-N\left|e^{\sum_{a=1,2} \sum_{i \in \mathbb{Z}} \alpha_{i}^{(a)} t_{i}^{(a)}} e^{\sum_{i, j} M_{i j} \psi_{i}^{(1)} \psi_{j}^{\dagger(2)}}\right| 0\right\rangle
\end{aligned}
$$

(see also Appendix A.2).

The tau function (153) is the tau function $\tau_{N, 0,0}^{(0)}$ of (140) where $g=$ $e^{\sum_{i, j} M_{i j} \psi_{i}^{(1)} \psi_{j}^{\dagger(2)}}$. This choice provides the semi-infinity of the TL equation which is

$$
\frac{\partial^{2} \tau_{N}^{\mathrm{TL}}}{\partial t_{1}^{(1)} \partial t_{-}^{(2)} 1} \tau_{N}^{\mathrm{TL}}-\frac{\partial \tau_{N}^{\mathrm{TL}}}{\partial t_{1}^{(1)}} \frac{\partial \tau_{N}^{\mathrm{TL}}}{\partial t_{1}^{(2)}}=-\tau_{N+1}^{\mathrm{TL}} \tau_{N-1}^{\mathrm{TL}}
$$

where we put $\tau_{N}^{\mathrm{TL}}\left(t^{(1)}, t^{(2)}\right)=\delta_{N, 0}$ for $N \leq 0$. Given $\tau_{1}^{\mathrm{TL}}$ one can construct all $\tau_{N}^{\mathrm{TL}}, N>1$ in a recursive way via (154).

Introduce

$$
e^{\sum_{k>0} z^{k} t_{k}}=: \sum_{k \geq 0} z^{k} s_{k}(t) \quad \text { and } s_{\{h\}}(t):=\operatorname{det}\left(s_{h_{i}-j}\right)_{i, j=1, \ldots, N}
$$

where $h=\left(h_{1}, \ldots, h_{N}\right), h_{1}>\cdots>h_{N} \geq 0$, and $s_{\{h\}}$ is the Schur function related to the partition $\lambda=\left(\lambda_{1}, \ldots, \lambda_{N}\right), \lambda_{i}=h_{i}+i-N$, see [19].

Using the relations

$$
\begin{aligned}
e^{\sum_{i \in \mathbb{Z}} \alpha_{i}^{(a)} t_{i}^{(a)}} \psi_{j}^{(a)} e^{-\sum_{i \in \mathbb{Z}} \alpha_{i}^{(a)} t_{i}^{(a)}} & =\sum_{k \geq 0} \psi_{j-k}^{(a)} s_{k}(t) \\
e^{\sum_{i \in \mathbb{Z}} \alpha_{i}^{(a)} t_{i}^{(a)}} \psi_{j}^{\dagger(a)} e^{-\sum_{i \in \mathbb{Z}} \alpha_{i}^{(a)} t_{i}^{(a)}} & =\sum_{k \geq 0} \psi_{j+k}^{\dagger(a)} s_{k}(-t)
\end{aligned}
$$

and Wick's rule (see [10]), the tau function (153) may be presented in the determinantal form:

$$
\tau_{N}^{\mathrm{TL}}\left(t^{(1)}, t^{(2)}\right)=\operatorname{det}\left(\mathbf{m}_{i j}\left(t^{(1)}, t^{(2)}\right)_{i, j=0, \ldots, N-1}\right.
$$

where

$$
\mathbf{m}_{i j}\left(t^{(1)}, t^{(2)}\right)=\sum_{k, l \geq 0} M_{i+k, j+l} s_{k}\left(t^{(1)}\right) s_{l}\left(-t^{(2)}\right)
$$

As we see $\tau_{1}^{\mathrm{TL}}=\mathbf{m}_{00}$.

The tau function (153) may also be written in Takasaki form [28,30,31] as

$$
\tau_{N}^{\mathrm{TL}}\left(t^{(1)}, t^{(2)}\right)=\sum_{\substack{h_{1}>\cdots>h_{N} \geq 0 \\ h_{1}^{\prime}>\cdots>h_{N}^{\prime} \geq 0}} M_{h, h^{\prime}} s_{\{h\}}\left(t^{(1)}\right) s_{\left\{h^{\prime}\right\}}\left(-t^{(2)}\right)
$$


where

$$
M_{\left\{h, h^{\prime}\right\}}:=\operatorname{det}\left[M_{h_{i}, h_{j}^{\prime}}\right]_{i, j=1}^{N}
$$

The series (157), where $M$ is specified, appears in various problems of random matrices, random partitions and in counting problems.

Another way to present tau functions of the semi-infinite TL (which is of use in many models of random matrices, see [12] and Examples in Appendix A.1 below) is

$$
\begin{aligned}
& \tau_{n, m}^{T L}\left(t^{(1)}, t^{(2)}, \bar{t}^{(1)}, \bar{t}^{(2)}\right) \\
& \quad=\left\langle n,-n\left|e^{\sum_{a=1,2} \sum_{i \in \mathbb{Z}} \alpha_{i}^{(a)} t_{i}^{(a)}} e^{\int \psi^{(1)}\left(z_{1}\right) \psi^{\dagger(2)}\left(z_{2}\right) \mathrm{d} \mu\left(z_{1}, z_{2}\right)} e^{\sum_{a=1,2} \sum_{i \in \mathbb{Z}} \alpha_{-i}^{(a)} \bar{t}_{i}^{(a)}}\right| m,-m\right\rangle
\end{aligned}
$$

where $\mathrm{d} \mu\left(z_{1}, z_{2}\right)$ is a bi-measure which should be specified according to the problem we are interested in. There are additional parameters here, $\bar{t}^{(1)}, \bar{t}^{(2)}$ and $m$, which are hidden parameters of the Toda lattice solutions. This is a particular case of the tau function (140).

From (159), we obtain $\tau_{n, m}^{T L}\left(t^{(1)}, t^{(2)}, \bar{t}^{(1)}, \bar{t}^{(2)}\right)=\delta_{n, m}$ for $n \leq m$.

Semi-infinite TL and semi-infinite B-type Pfaff Lattice (BPL).

PROPOSITION 4.

$$
\left(\tau_{N}^{B}(t)\right)^{2}=\tau_{N}^{\mathrm{TL}}(t,-t)
$$

where

$$
\begin{aligned}
& \tau_{N}^{B}(t)=\left\langle N\left|e^{\sum_{i \in \mathbb{Z}} \beta_{i} t_{i}} e^{\frac{1}{2} \sum_{i, j} A_{i j} \psi_{i} \psi_{j}+\sqrt{2} \sum_{i} a_{i} \psi_{i} \varphi} e^{\sum_{i \in \mathbb{Z}} \beta_{-i} \bar{t}_{i}}\right| 0\right\rangle \\
& \tau_{N}^{\mathrm{TL}}(t,-t)=(-)^{\frac{N(N+1)}{2}}\left\langle N,-N\left|e^{\sum_{i \in \mathbb{Z}}\left(\alpha_{i}^{(1)}-\alpha_{i}^{(2)}\right) t_{i}} e^{\sum_{i, j}\left(A_{i j}-a_{i} a_{j}\right) \psi_{i}^{(1)} \psi^{\dagger(2)}}\right| 0\right\rangle
\end{aligned}
$$

and $A_{i j}=-A_{j i}$.

It is well-known that the determinant of a skew symmetric $N \times N$ matrix vanishes if $N$ is odd. The square root of a skew symmetric matrix of even size is the Pfaffian (see Appendix A.5) of this matrix. Let us call the sum of a skew symmetric matrix and a symmetric matrix of rank 1 the quasi-skew symmetric matrix. The square root of a quasi-skew symmetric matrix may be identified with a Pfaffian of some different matrix:

LEMMA 2. The square root of the determinant of the quasi-skew symmetric $N \times N$ matrix with entries $A_{i j}-a_{i} a_{j}, i, j=1, \ldots, N$, where $A_{i j}=-A_{j i}$, is the Pfaffian of the $2\left[\frac{N+1}{2}\right] \times 2\left[\frac{N+1}{2}\right]$ matrix $B$ which is defined as follows. For $N$ even, $B=A$. For $N$ odd, $N=2 n-1$ and

$$
\mathrm{B}_{i j}=-\mathrm{B}_{j i}:=\left\{\begin{array}{lll}
A_{i, j} & \text { if } & 1 \leq i<j \leq 2 n-1 \\
a_{i} & \text { if } & 1 \leq i<j=2 n
\end{array} .\right.
$$


Thus, for both odd and even $N$, we have

$$
(\operatorname{Pf} B)^{2}=\operatorname{det}\left(A_{i, j}-a_{i} a_{j}\right)_{i, j=1, \ldots, N}
$$

We have the following

\section{COROLLARY 1.}

$$
\begin{aligned}
& \left(\sum_{h_{1}>\cdots>h_{N}} \sqrt{\operatorname{det}\left(A_{h_{i}, h_{j}}-a_{h_{i}} a_{h_{j}}\right)} s_{\{h\}}(t)\right)^{2} \\
& =\sum_{\substack{h_{1}>\cdots>h_{N} \\
h_{1}^{\prime}>\cdots>h_{N}^{\prime}}} \operatorname{det}\left(A_{h_{i}, h_{j}^{\prime}}-a_{h_{i}} a_{h_{j}^{\prime}}\right) s_{\{h\}}(t) s_{\left\{h^{\prime}\right\}}(t)
\end{aligned}
$$

Similarly, we have the following

\section{PROPOSITION 5.}

$$
\left(\tau_{n, m}^{B}(t, \bar{t})\right)^{2}=\tau_{n, m}^{T L}(t,-t ; \bar{t},-\bar{t})
$$

where

$$
\begin{aligned}
& \tau_{n, m}^{B}(t, \bar{t})=\left\langle n\left|e^{\sum_{i \in \mathbb{Z}} \beta_{i} t_{i}} e^{\frac{1}{2} \int \psi\left(z_{1}\right) \psi\left(z_{2}\right) \mathrm{d} \omega\left(z_{1}, z_{2}\right)+\sqrt{2} \int \psi(z) \mathrm{d} v(z) \varphi} e^{\sum_{i \in \mathbb{Z}} \beta_{-i} \bar{t}_{i}}\right| m\right\rangle \\
& (-)^{\frac{n(n+1)}{2}+\frac{m(m+1)}{2}} \tau_{n, m}^{\mathrm{TL}}(t,-t ; \bar{t},-\bar{t})= \\
& \left\langle n,-n\left|e^{\sum_{i \in \mathbb{Z}}\left(\alpha_{i}^{(1)}-\alpha_{i}^{(2)}\right) t_{i}} e^{\int \psi^{(1)}\left(z_{1}\right) \psi^{\dagger(2)}\left(z_{2}\right)\left(\mathrm{d} \omega\left(z_{1}, z_{2}\right)-\mathrm{d} v\left(z_{1}\right) \mathrm{d} v\left(z_{2}\right)\right)} e^{\sum_{i \in \mathbb{Z}}\left(\alpha_{-i}^{(1)}-\alpha_{-i}^{(2)}\right) \bar{t}_{i}}\right| m,-m\right\rangle
\end{aligned}
$$

Here, $\mathrm{d} \omega\left(z_{1}, z_{2}\right)=-\mathrm{d} \omega\left(z_{1}, z_{2}\right)$ and $\mathrm{d} v(z)$ are measures.

The first applications of BKP tau functions (167) were given in [16] to describe orthogonal and symplectic ensembles of random matrices. In Appendix A.1, one may find further examples.

\section{Outlook}

In our next paper [18], the analog of the Proposition 1 for the multicomponent BKP tau functions [15] will be written down. Also, we shall consider the relations between various matrix integrals and between series over partitions which result from Propositions 1 and 5. 


\section{Acknowledgements}

One of the authors (A.O.) thanks K.Takasaki and T.Shiota for helpful discussions regarding Toda lattice and Pfaff lattice. He (A.O.) is supported by RFBR Grant 14-01-00860.

Open Access This article is distributed under the terms of the Creative Commons Attribution 4.0 International License (http://creativecommons.org/licenses/by/4.0/), which permits unrestricted use, distribution, and reproduction in any medium, provided you give appropriate credit to the original author(s) and the source, provide a link to the Creative Commons license, and indicate if changes were made.

\section{A. Appendices}

\section{A.1. APPLICATIONS OF TWO-SIDED 2-KP AND TWO-SIDED BKP HIERARCHIES TO MATRIX MODELS}

(I). First, we recall some not very well-known known results concerning applications of the two-sided 2-KP tau functions written in form (159) to a number of matrix models:

EXAMPLE 1.1. The choice $\mathrm{d} \mu\left(z_{1}, z_{2}\right)=e^{-|z|^{2}} \delta^{(2)}\left(z_{2}-\bar{z}_{1}\right) \mathrm{d}^{2} z_{1} \mathrm{~d}^{2} z_{2}, z_{1,2} \in \mathbb{C}$ yields (see [12]) both the fermionic representation for the partition function of the ensemble of normal matrices (about this ensemble see [3] and [21]), and the partition function of the complex Ginibre ensemble (about Ginibre ensemble of complex matrices see Ch. 15.1 in [20]). In this example $\bar{z}$ is the complex conjugate of $z$. Here $\delta^{(2)}$ is the two-dimensional delta function.

EXAMPLE 1.2. The choice $\mathrm{d} \mu\left(z_{1}, z_{2}\right)=e^{z_{1} z_{2}} \mathrm{~d} z_{1} \mathrm{~d} z_{2}, z_{1,2} \in \mathbb{R}$ yields (see [12]) the fermionic representation for the partition function of the two-matrix model introduced in [9] (the relation of the two-matrix model and TL see in [6] and different fermionic representation see in [7]). If we take $z_{1,2} \in S^{1}$, we obtain the model of two unitary matrices $[35,36]$.

EXAMPLE 1.3. The choice $\mathrm{d} \mu\left(z_{1}, z_{2}\right)=\delta\left(z_{1}-z_{2}\right) \mathrm{d} z_{1} \mathrm{~d} z_{2}, z_{1,2} \in \mathbb{R}$ yields (see [8]) the fermionic representation for the partition function of the one-matrix model (for the relation of the one-matrix model, the one-dimensional TL see in [6] and different fermionic representation see [7]).

EXAMPLE 1.4. Take $z_{a}=e^{i \phi_{a}}, a=1,2$. The choice $\mathrm{d} \mu\left(e^{i \phi_{1}}, e^{i \phi_{2}}\right)=\delta\left(\phi_{1}-\phi_{2}\right) \mathrm{d} \phi_{1} \mathrm{~d} \phi_{2}$, $0 \leq \phi_{1}, \phi_{2}, \phi \leq 2 \pi$, yields the fermionic representation for the $\beta=2$ circlar ensemble (about this ensemble see Ch. 10.3 in [20] and [22]). 
In case the bi-measure $\mathrm{d} \mu$ and the matrix $M$ are related by the moment's transform

$$
M_{i j}=M_{i j}\left(\bar{t}^{(1)}, \bar{t}^{(2)}, m\right)=\int z_{1}^{i+m} z_{2}^{j+m} e^{\sum_{k \geq 0}\left(\bar{t}_{k}^{(1)} z_{1}^{-k}-\bar{t}_{k}^{(2)} z_{2}^{-k}\right)} \mathrm{d} \mu\left(z_{1}, z_{2}\right)
$$

the tau functions (153) and (153) may be equated as follows:

$$
\tau_{N}^{2 \mathrm{KP}}\left(t^{(1)}, t^{(2)} ; \bar{t}^{(1)}, \bar{t}^{(2)}, m\right)=e^{\sum_{a=1,2} \sum_{k \geq 1} k t_{k}^{(a)} \bar{t}_{k}^{(a)}} \tau_{N+m, m}^{T L}\left(t^{(1)}, t^{(2)}, \bar{t}^{(1)}, \bar{t}^{(2)}\right)
$$

Now the tau function (153) written here depends on the additional parameters $\bar{t}^{(1)}, \bar{t}^{(2)}, m$. The expression for $\tau_{1}^{\mathrm{TL}}$ :

$$
\begin{aligned}
\tau_{m+1, m}^{T L}\left(t^{(1)}, t^{(2)}, \bar{t}^{(1)}, \bar{t}^{(2)}\right) & =c(t) \int z_{1}^{m} z_{2}^{m} e^{\sum_{i \geq 1}\left(z_{1}^{i} t_{i}^{(1)}-z_{2}^{i} t_{i}^{(2)}-z_{1}^{-i} \bar{t}_{i}^{(1)}+z_{2}^{-i} \bar{t}_{i}^{(2)}\right)} \mathrm{d} \mu\left(z_{1}, z_{2}\right) \\
c(t) & =e^{\sum_{i \geq 1}\left(i t_{i}^{(1)} \bar{t}_{i}^{(1)}+i t_{i}^{(2)} \bar{t}_{i}^{(1)}\right)}
\end{aligned}
$$

allows to obtain all $\tau_{N}^{\mathrm{TL}}, N>1$, in the recursive way from the TL Hirota equation (154).

(II). For applications of the BKP tau functions (167), we present a set of yet unpublished results taken from [27]:

EXAMPLE 2.1. The choice $\mathrm{d} \omega\left(z_{1}, z_{2}\right)=\frac{1}{2} \operatorname{sgn}\left(z_{1}-z_{2}\right) \mathrm{d} z_{1} \mathrm{~d} z_{2}$ and $\mathrm{d} v(z)=\mathrm{d} z$, $z_{1,2}, z \in \mathbb{R}$, yields the fermionic representation for the $\beta=1$ ensemble (orthogonal ensemble, see Ch. 7 in [20]) [16].

EXAMPLE 2.2. The choice $\mathrm{d} \omega\left(z_{1}, z_{2}\right)=\frac{1}{4}\left(\partial_{z_{1}}-\partial_{z_{2}}\right) \delta\left(z_{1}-z_{2}\right) \mathrm{d} z_{1} \mathrm{~d} z_{2}$ and $\mathrm{d} v(z)=$ $0, z_{1,2} \in \mathbb{R}$, yields the fermionic representation for the $\beta=4$ ensemble (symplectic ensemble, see Ch. 8 in [20]) [16].

EXAMPLE 2.3. Take $z_{a}=e^{i \phi_{a}}$. The choice $\mathrm{d} \omega\left(e^{i \phi_{1}}, e^{i \phi_{2}}\right)=\frac{1}{2} \operatorname{sgn}\left(\phi_{1}-\phi_{2}\right) \mathrm{d} \phi_{1} \mathrm{~d} \phi_{2}$ and $\mathrm{d} v\left(e^{i \phi}\right)=\mathrm{d} \phi, 0 \leq \phi_{1}, \phi_{2}, \phi \leq 2 \pi$. This yields (see [26]) the fermionic representation for the $\beta=1$ circular ensemble (about this ensemble see Ch. 10.1 in [20]).

EXAMPLE 2.4. The choice $\mathrm{d} \omega\left(e^{i \phi_{1}}, e^{i \phi_{2}}\right)=\frac{1}{4}\left(\partial_{\phi_{1}}-\partial_{\phi_{2}}\right) \delta\left(\phi_{1}-\phi_{2}\right) \mathrm{d} \phi_{1} \mathrm{~d} \phi_{2}$ and $\mathrm{d} v\left(e^{i \phi}\right)=\mathrm{d} \phi, 0 \leq \phi_{1}, \phi_{2}, \phi \leq 2 \pi$, yields (see [26]) the fermionic representation for the $\beta=4$ circular ensemble (about this ensemble see Ch. 10.2 in [20]).

EXAMPLE 2.5. The choice $\mathrm{d} \omega\left(z_{1}, z_{2}\right)=\delta\left(z_{1}+z_{2}\right) \mathrm{d} z_{1} \mathrm{~d} z_{2}$ and $\mathrm{d} \nu(z)=\delta(z) \mathrm{d} z$, $z_{1,2}, z \in \mathbb{R}$, yields (see [26]) the fermionic representation for the ensemble of antisymmetric matrices (see Ch. 13 in [20] about this ensemble).

EXAMPLE 2.6. For the Pandey-Mehta interpolating ensembles, $0<\alpha^{2}<\infty$ being the interpolation parameter, see [20] Ch. 14 
(a) for the ensemble interpolating between GUE and GOE, see [20] Ch. 14.1, we take

$$
\begin{gathered}
\mathrm{d} \omega\left(z_{1}, z_{2}\right)=e^{-\frac{1}{2}\left(1+\alpha^{2}\right)\left(z_{1}^{2}+z_{2}^{2}\right)} \operatorname{erf}\left(\sqrt{\frac{1-\alpha^{4}}{4 \alpha^{2}}}\left(z_{1}-z_{2}\right)\right) \mathrm{d} z_{1} \mathrm{~d} z_{2}, \\
\mathrm{~d} v(z)=e^{-\frac{1}{2}\left(1+\alpha^{2}\right) z^{2}} \mathrm{~d} z, \quad z_{1,2}, z \in \mathbb{R}
\end{gathered}
$$

Here $\operatorname{erf}(x)=\int_{0}^{x} e^{-x^{2}} \mathrm{~d} x$.

(b) for the ensemble interpolating between GUE and GSE, see [20] Ch. 14.2, we take

$$
\mathrm{d} \omega\left(z_{1}, z_{2}\right)=\left(z_{1}-z_{2}\right) e^{-\frac{1}{2}\left(1+\alpha^{2}\right)\left(z_{1}^{2}+z_{2}^{2}\right)} e^{-\frac{\left(1-\alpha^{4}\right)\left(z_{1}-z_{2}\right)^{2}}{4 \alpha^{2}}} \mathrm{~d} z_{1} \mathrm{~d} z_{2}, \quad z_{1,2} \in \mathbb{R}
$$

The same measures $\mathrm{d} \omega, \mathrm{d} v$ are available to get the asymmetric two-matrix model where the first matrix is Hermitian while the second is respectively (a) either symmetric $\left(0<\alpha^{2}<1\right)$, or antisymmetric $\left(1<\alpha^{2}<\infty\right)$ (b) either self-dual $\left(0<\alpha^{2}<1\right)$, or anti-self-dual $\left(1<\alpha^{2}<\infty\right)$, see [26].

EXAMPLE 2.7. The choice $\mathrm{d} \omega\left(z_{1}, z_{2}\right)=\frac{1}{2}(\bar{z}-z) e^{-|z|^{2}} \delta^{(2)}\left(z_{2}-\bar{z}_{1}\right) \mathrm{d}^{2} z_{1} \mathrm{~d}^{2} z_{2}, z_{1,2} \in \mathbb{C}$ and $\mathrm{d} v(z)=0$ yields (see [24]) the fermionic representation for the ensemble of random (non-Hermitian) quaternionic matrices (the so-called quaternion-real Ginibre ensemble (see Ch. 15.2 in [20]). Here $\delta^{(2)}$ is the two-dimensional delta function. In this example $\bar{z}$ is the complex conjugate of $z$.

EXAMPLE 2.8. For the Ginibre ensemble of real matrices (see Ch. 15.3 in [20]) the choice of the measure is more complicated, and may be found in [24].

EXAMPLE 2.9. The choice $\mathrm{d} \omega\left(z_{1}, z_{2}\right)=\frac{1}{2} \frac{z_{1}-z_{2}}{z_{1}+z_{2}} \mathrm{~d} z_{1} \mathrm{~d} z_{2}, z_{1,2} \in \mathbb{R}$, yields (see [26]) the fermionic representation for the so-called Bures ensembles which appears in quantum chaos problems where random density matrix appears [11] and [23].

EXAMPLE 2.10. The choice $\mathrm{d} \omega\left(z_{1}, z_{2}\right)=\frac{1}{2} \tanh \pi\left(z_{1}-z_{2}\right) \mathrm{d} z_{1} \mathrm{~d} z_{2}, z_{1,2} \in \mathbb{R}$ yields (see [26]) the fermionic representation for the Plancheral measure for the group $S L(N, \mathbb{R})$ (and for the symmetric space $S L(N, \mathbb{R}) / S O(N)$ ), see Section 17.2.8 in [33] where put $\lambda_{i}=\frac{1}{2} z_{i}$.

EXAMPLE 2.11. The choice $\mathrm{d} \omega\left(z_{1}, z_{2}\right)=\frac{1}{2 \pi i}\left(z_{1}-z_{1}^{-1}\right) \delta\left(z_{2}-z_{1}^{-1}\right) \mathrm{d} z_{1} \mathrm{~d} z_{2}$ and $\mathrm{d} v(z)=0, z_{1,2}, z \in S^{1}$ yields the ensemble of symplectic matrices, details will be published in the forthcoming paper [18]. 
EXAMPLE 2.12. The choice $\mathrm{d} \omega\left(z_{1}, z_{2}\right)=\frac{1}{2 \pi i}\left(z_{2}^{-1}-z_{2}\right)^{-1} \delta\left(z_{2}-z_{1}^{-1}\right) \mathrm{d} z_{1} \mathrm{~d} z_{2}$ and $\mathrm{d} v(z)=\frac{1}{2} \delta(z-1) \mathrm{d} z, z_{1,2}, z \in S^{1}$ yields the ensemble of orthogonal matrices, for details we refer to the forthcoming paper [18].

The relation between realizations (161) and (167) is given by

$$
\begin{aligned}
A_{i j} & =A_{i j}(\bar{t}, m)=\int z_{1}^{i+m} z_{2}^{j+m} e^{\sum_{k \geq 0} \bar{t}_{k}\left(z_{1}^{-k}+z_{2}^{-k}\right)} \mathrm{d} \omega\left(z_{1}, z_{2}\right), \\
a_{i} & =a_{i}(\bar{t}, m)=\int z^{i+m} e^{\sum_{k \geq 0} \bar{t}_{k} z^{-k}} \mathrm{~d} v(z)
\end{aligned}
$$

In $B$-case relations (155),(156) read as

$$
\begin{aligned}
\tau_{N}^{\mathrm{TL}}(t,-t ; \bar{t},-\bar{t}, m)= & \operatorname{det}\left(\mathbf{m}_{i j}(t ; \bar{t}, m)\right)_{i, j=0, \ldots, N-1} \\
\mathbf{m}_{i j}(t ; \bar{t}, m)= & \int z_{1}^{i+m} z_{2}^{j+m} e^{\sum_{k \geq 1}\left(z_{1}^{k}+z_{2}^{k}\right) t_{k}-\left(z_{1}^{-k}+z_{2}^{-k}\right) \bar{t}_{k}}\left(\mathrm{~d} \omega\left(z_{1}, z_{2}\right)\right. \\
& \left.-\mathrm{d} v\left(z_{1}\right) d v\left(z_{1}\right)\right) \\
= & \sum_{k, l \geq 0} A_{i+k, j+l}(\bar{t}, m) s_{k}(t) s_{l}(t) \\
& -\sum_{k \geq 0} a_{i+k}(\bar{t}, m) s_{k}(t) \sum_{l \geq 0} a_{j+l}(\bar{t}, m) s_{l}(t)
\end{aligned}
$$

As we see the matrix $\mathbf{m}$ is quasi-skew symmetric.

Then, for both odd and even $N$, we have from Proposition 5

$$
\left(\tau_{N}^{B}(t ; \bar{t}, m)\right)^{2}=\operatorname{det}\left(\mathbf{m}_{i j}(t ; \bar{t}, m)\right)_{i, j=0, \ldots, N-1}
$$

According to Lemma 2, $\tau_{N}^{B}$ is a certain Pfaffian (this result may be obtained also from the Wick theorem applied to the fermionic expectation value (167).

We obtain a generalization of the result presented in [1], [2] where the relation (176) was achieved for the case of even $N$ and for skew symmetric matrices $\mathbf{m}$. This case may referred as the $D$-type Pfaff lattice (DPL), see [29].

\section{A.2 HIROTA EQUATION FOR THE TL AND FOR THE 2-COMPONENT KP TAU} FUNCTIONS.

The TL tau function was introduced in [10] and may be defined by

$$
\tau_{n}^{\mathrm{TL}}(t, \bar{t})=\left\langle n\left|e^{\sum_{i>0} t_{i} \alpha_{i}} g^{\mathrm{TL}} e^{-\sum_{i>0} \bar{t}_{i} \alpha_{-i}}\right| n\right\rangle
$$

This tau function solves Hirota equation, [10,32]

$$
\begin{aligned}
& \oint \frac{\mathrm{d} z}{2 \pi i} z^{n^{\prime}-n} e^{V\left(t^{\prime}-t, z\right)} \tau_{n^{\prime}}^{\mathrm{TL}}\left(t^{\prime}-\left[z^{-1}\right], \bar{t}^{\prime}\right) \tau_{n}^{\mathrm{TL}}\left(t+\left[z^{-1}\right], \bar{t}\right) \\
& \quad \oint \frac{\mathrm{d} z}{2 \pi i} z^{n^{\prime}-n} e^{V\left(\bar{t}^{\prime}-\bar{t}, z^{-1}\right)} \tau_{n^{\prime}+1}^{\mathrm{TL}}\left(t^{\prime}, \bar{t}^{\prime}-[z]\right) \tau_{n-1}^{\mathrm{TL}}(t, \bar{t}+[z])
\end{aligned}
$$


(see $[10,32])$ which includes

$$
\frac{\partial^{2} \tau_{n}^{\mathrm{TL}}}{\partial t_{1} \partial \bar{t}_{1}} \tau_{n}^{\mathrm{TL}}-\frac{\partial \tau_{n}^{\mathrm{TL}}}{\partial t_{1}} \frac{\partial \tau_{n}^{\mathrm{TL}}}{\partial \bar{t}_{1}}=-\tau_{n+1}^{\mathrm{TL}} \tau_{n-1}^{\mathrm{TL}}
$$

The two-component KP tau function

$$
\tau_{n}^{2 \mathrm{KP}}(t, \bar{t})=\left\langle n,-n\left|e^{\sum_{i>0}\left(t_{i}^{\prime} \alpha_{i}^{(1)}+\bar{t}_{i}^{\prime} \alpha_{i}^{(2)}\right)} g^{2 \mathrm{KP}}\right| 0\right\rangle
$$

solves Hirota equation

$$
\begin{aligned}
& \oint \frac{\mathrm{d} z}{2 \pi i}(-)^{-n^{\prime}-n} z^{n^{\prime}-n} e^{V\left(t^{\prime}-t, z\right)} \tau_{n^{\prime}}^{2 \mathrm{KP}}\left(t^{\prime}-\left[z^{-1}\right], \bar{t}^{\prime}\right) \tau_{n}^{2 \mathrm{KP}}\left(t+\left[z^{-1}\right], \bar{t}\right) \\
& =\oint \frac{\mathrm{d} z}{2 \pi i} z^{n-n^{\prime}-2} e^{V\left(\bar{t}^{\prime}-\bar{t}, z\right)} \tau_{n^{\prime}+1}^{2 \mathrm{KP}}\left(t^{\prime}, \bar{t}^{\prime}-\left[z^{-1}\right]\right) \tau_{n-1}^{2 \mathrm{KP}}\left(t, \bar{t}+\left[z^{-1}\right]\right)
\end{aligned}
$$

which up to the sign factor $(-)^{n+n^{\prime}}$ in the first integral is equal to (178) if we change $z \rightarrow z^{-1}$ in the second integral in (181). This brings us to the relation (152).

\section{A.3 HIROTA EQUATION FOR THE TWO-SIDED TWO-COMPONENT KP.}

The two-sided two-component KP tau function (140)

$$
\begin{aligned}
& \tau_{N}\left(L^{(1)}, L^{(2)} ; t^{(1)}, t^{(2)} ; \bar{t}^{(1)}, \bar{t}^{(1)}\right) \\
& \quad=\left\langle N+L^{(1)},-N+L^{(2)}\right| e^{\sum_{k=1}^{\infty}\left(t_{k}^{(1)} \alpha_{k}^{(1)}+t_{k}^{(2)} \alpha_{k}^{(1)}\right) g e^{\sum_{k=1}^{\infty}\left(\bar{t}_{-k}^{(1)} \alpha_{-k}^{(1)}+\bar{t}_{k}^{(2)} \alpha_{-k}^{(2)}\right)}\left|L^{(1)}, L^{(2)}\right\rangle}
\end{aligned}
$$

solves the following Hirota equations which tell that the residium of

$$
\begin{aligned}
& (-z)^{N^{\prime}-N+L^{(1) \prime}-L^{(1)}} e^{V\left(t^{(1) \prime}-t^{(1)}, z\right)} \tau_{N^{\prime}}^{(0)}\left(L^{(1) \prime}, L^{(2) \prime} ; t_{-}^{(1) \prime}(z), t^{(2) \prime} ; \overline{\mathbf{t}}^{\prime}\right) \\
& \times \tau_{N}^{(0)}\left(L^{(1)}, L^{(2)} ; t_{+}^{(1)}(z), t^{(2)} ; \overline{\mathbf{t}}\right) \\
& +z^{N-N^{\prime}+L^{(2)^{\prime}}-L^{(2)}-2} e^{V\left(t^{(2) \prime}-t^{(2)}, z\right)} \tau_{N^{\prime}+1}^{(0)}\left(L^{(1) \prime}, L^{(2) \prime} ; t^{(1) \prime}, t_{-}^{(2) \prime}(z) ; \overline{\mathbf{t}}^{\prime}\right) \\
& \times \tau_{N-1}^{(0)}\left(L^{(1)}, L^{(2)} ; t^{(1)}, t_{+}^{(2)}(z) ; \overline{\mathbf{t}}\right) \\
& -(-z)^{L^{(1) \prime}-L^{(1)}} e^{V\left(\bar{t}^{(1)}-\bar{t}^{(1) \prime}, z^{-1}\right)} \tau_{N^{\prime}}^{(0)}\left(L_{+}^{(1) \prime}, L^{(2) \prime} ; \mathbf{t}^{\prime}, \bar{t}_{+}^{(1) \prime}(z), \bar{t}^{(2) \prime}\right) \\
& \times \tau_{N}^{(0)}\left(L_{-}^{(1)}, L^{(2)} ; \mathbf{t} ; \bar{t}_{-}^{(1)}(z), \bar{t}^{(2)}\right) \\
& -z^{L^{(2) \prime}-L^{(2)}} e^{V\left(\bar{t}^{(2)}-\bar{t}^{(2) \prime}, z^{-1}\right)} \tau_{N^{\prime}+1}^{(0)}\left(L^{(1) \prime}, L_{+}^{(2) \prime} ; \mathbf{t}^{\prime} ; \bar{t}^{(1) \prime}, \bar{t}_{+}^{(2) \prime}(z)\right) \\
& \times \tau_{N-1}^{(0)}\left(L^{(1)}, L_{-}^{(2)} ; \mathbf{t} ; \bar{t}^{(1)}, \bar{t}_{-}^{(2)}(z)\right)
\end{aligned}
$$


vanishes. Here $\mathbf{L}^{\prime}=\left(L^{(1) \prime}, L^{(2) \prime}\right)$ and $\mathbf{L}=\left(L^{(1)}, L^{(2)}\right)$ are pairs of integers, and $\mathbf{t}^{\prime}=\left(\bar{t}^{(1) \prime}, \bar{t}^{(2) \prime}\right), \mathbf{t}^{\prime}=\left(\bar{t}^{(1)}, \bar{t}^{(2)}\right), \bar{t}^{(a) \prime}=\left(t_{1}^{(2)}, t_{2}^{(a)}, \ldots\right), \bar{t}^{(a) \prime}=\left(\bar{t}_{1}^{(2) \prime}, \bar{t}_{2}^{(a) \prime}, \ldots\right)$ are semiinfinite sets of higher times. Then

$$
\begin{aligned}
t_{ \pm}^{(a) \prime}(z):= & t^{(a) \prime} \pm\left[z^{-1}\right], \quad \bar{t}_{ \pm}^{(a) \prime}(z):=\bar{t}^{(a) \prime} \pm[z], \quad t_{ \pm}^{(a)}(z):=t^{(a)} \pm\left[z^{-1}\right], \\
& \bar{t}_{ \pm}^{(a)}(z):=\bar{t}^{(a)} \pm[z],
\end{aligned}
$$

and

$$
L_{ \pm}^{(a) \prime}=L^{(a) \prime} \pm 1, \quad L_{ \pm}^{(a)}=L^{(a)} \pm 1
$$

where $a=1,2$.

\section{A.4 HIROTA EQUATIONS FOR THE TWO-SIDED BKP.}

Hirota equations for the large BKP hierarchy were written in [15]. For the twosided BKP hierarchy (2-BKP hierarchy) (141), Hirota equations are as follows [25]

$$
\begin{aligned}
\oint & \frac{\mathrm{d} z}{2 \pi i} z^{N^{\prime}+L^{\prime}-N-L-2} e^{V\left(s^{\prime}-s, z\right)} \tau_{N^{\prime}-1}\left(L^{\prime}, s^{\prime}-\left[z^{-1}\right], \bar{s}^{\prime}\right) \tau_{N+1}\left(L, s+\left[z^{-1}\right], \bar{s}\right) \\
& +\oint \frac{\mathrm{d} z}{2 \pi i} z^{N+L-N^{\prime}-L^{\prime}-2} e^{V\left(s-s^{\prime}, z\right)} \tau_{N^{\prime}+1}\left(L^{\prime}, s^{\prime}+\left[z^{-1}\right], \bar{s}^{\prime}\right) \tau_{N-1}\left(L, s-\left[z^{-1}\right], \bar{s}\right) \\
= & \oint \frac{\mathrm{d} z}{2 \pi i} z^{L^{\prime}-L} e^{V\left(\bar{s}^{\prime}-\bar{s}, z^{-1}\right)} \tau_{N^{\prime}-1}\left(L^{\prime}+1, s^{\prime}, \bar{s}^{\prime}-[z]\right) \tau_{N+1}(L-1, s, \bar{s}-[z]) \\
& +\oint \frac{\mathrm{d} z}{2 \pi i} z^{L-L^{\prime}} e^{V\left(\bar{s}^{\prime}-\bar{s}, z^{-1}\right)} \tau_{N^{\prime}+1}\left(L^{\prime}-1, s^{\prime}, \bar{s}^{\prime}+[z]\right) \tau_{N-1}(L+1, s, \bar{s}+[z]) \\
& +\frac{(-1)^{L^{\prime}+L}}{2}\left(1-(-1)^{N^{\prime}+N}\right) \tau_{N^{\prime}}\left(L^{\prime}, s^{\prime}, \bar{s}^{\prime}\right) \tau_{N}(L, s, \bar{s})
\end{aligned}
$$

The difference BKP Hirota equation may be obtained from the previous one, see [25]

$$
\begin{aligned}
- & \frac{\beta}{\alpha-\beta} \tau_{N}\left(l, \mathbf{t}+\left[\beta^{-1}\right]\right) \tau_{N+1}\left(l, \mathbf{t}+\left[\alpha^{-1}\right]\right)-\frac{\alpha}{\beta-\alpha} \tau_{N}\left(l, \mathbf{t}+\left[\alpha^{-1}\right]\right) \tau_{N+1}\left(l, \mathbf{t}+\left[\beta^{-1}\right]\right) \\
& +\frac{1}{\alpha \beta} \tau_{N+2}\left(l, \mathbf{t}+\left[\alpha^{-1}\right]+\left[\beta^{-1}\right]\right) \tau_{N-1}(l, \mathbf{t})=\tau_{N+1}\left(l, \mathbf{t}+\left[\alpha^{-1}\right]+\left[\beta^{-1}\right]\right) \tau_{N}(l, \mathbf{t}) .
\end{aligned}
$$


By replacing $z \rightarrow z^{-1}$ in the last two members of (184), it may be written as follows

$$
\begin{aligned}
& \oint \frac{\mathrm{d} z}{2 \pi i} z^{N^{\prime}+L^{\prime}-N-L-2} e^{V\left(s^{\prime}-s, z\right)} \tau_{N^{\prime}-1}\left(L^{\prime}, s^{\prime}-\left[z^{-1}\right], \bar{s}^{\prime}\right) \tau_{N+1}\left(L, s+\left[z^{-1}\right], \bar{s}\right) \\
& \quad+\oint \frac{d z}{2 \pi i} z^{N+L-N^{\prime}-L^{\prime}-2} e^{V\left(s-s^{\prime}, z\right)} \tau_{N^{\prime}+1}\left(L^{\prime}, s^{\prime}+\left[z^{-1}\right], \bar{s}^{\prime}\right) \tau_{N-1}\left(L, s-\left[z^{-1}\right], \bar{s}\right) \\
& \quad-\oint \frac{\mathrm{d} z}{2 \pi i} z^{L-L^{\prime}-2} e^{V\left(\bar{s}^{\prime}-\bar{s}, z\right)} \tau_{N^{\prime}-1}\left(L^{\prime}+1, s^{\prime}, \bar{s}^{\prime}-\left[z^{-1}\right]\right) \tau_{N+1}\left(L-1, s, \bar{s}-\left[z^{-1}\right]\right) \\
& \quad-\oint \frac{\mathrm{d} z}{2 \pi i} z^{L^{\prime}-L-2} e^{V\left(\bar{s}^{\prime}-\bar{s}, z\right)} \tau_{N^{\prime}+1}\left(L^{\prime}-1, s^{\prime}, \bar{s}^{\prime}+\left[z^{-1}\right]\right) \tau_{N-1}\left(L+1, s, \bar{s}+\left[z^{-1}\right]\right) \\
& =\frac{(-1)^{L^{\prime}+L}}{2}\left(1-(-1)^{N^{\prime}+N}\right) \tau_{N^{\prime}}\left(L^{\prime}, s^{\prime}, \bar{s}^{\prime}\right) \tau_{N}(L, s, \bar{s})
\end{aligned}
$$

which is up to the sign factor is the Hirota equation for the two-component BKP [15] tau function:

$$
\tau\left(N^{(1)}, N^{(2)} ; s^{(1)}, s^{(2)}\right):=(-)^{\frac{N^{(2)}\left(N^{(2)}+1\right)}{2}}\left\langle N^{(1)}, N^{(2)}\left|e^{\sum_{a=1,2} \sum_{i>0} \beta^{a} s^{(a)}} h^{(1,2)}\right| 0,0\right\rangle
$$

\section{A.5 PFAFFIANS}

The determinant of an anti-symmetric matrix $A$ of odd order vanishes. For even order, say $k$, the following multilinear form in $A_{i j}, i<j \leq k$

$$
\operatorname{Pf}[A]:=\sum_{\sigma} \operatorname{sgn}(\sigma) A_{\sigma(1), \sigma(2)} A_{\sigma(3), \sigma(4)} \ldots A_{\sigma(k-1), \sigma(k)}
$$

where sum runs over all permutation restricted by

$$
\sigma: \sigma(2 i-1)<\sigma(2 i), \quad \sigma(1)<\sigma(3)<\cdots<\sigma(k-1),
$$

coincides with the square root of $\operatorname{det} A$ and is called the Pfaffian of $A$, see, for instance [20].

\section{References}

1. Adler, M., van Moerbeke, P.: Symmetric random matrices and the Pfaff lattice. arXiv:solv-int/9903009v1

2. Adler, M., van Moerbeke, P., Shiota, T.: Pfaff $\tau$-functions. Math. Ann. 322, 423-476 (2002). arXiv:nlin/9909010

3. Chau, L.-L., Zaboronsky, O.: On the structure of correlation functions in the normal matrix models. Commun. Math. Phys. 196, 203-247 (1998)

4. Date, E., Jimbo, M., Kashiwara, M., Miwa, T.: Transformation groups for soliton equations. In: Jimbo, M., Miwa, T. (eds.) Nonlinear integrable systems - classical theory and quantum theory, pp. 39-120. World Scientific (1983)

5. Date, E., Jimbo, M., Kashiwara, M., Miwa, T.: Transformation groups for soliton equations, IV. A new hierarchy of soliton equations of KP-type. Physica D 4, 343365 (1982) 
6. Gerasimov, A., Marshakov, A., Mironov, A., Morozov, A., Orlov, A.: Matrix models of two-dimensional gravity and Toda theory. Nucl. Phys. B 357(2-3), 565-618 (1991)

7. Kharchev, K., Marshakov, A., Mironov, A., Orlov, A., Zabrodin, A.: Matrix models among integrable theories: Forced hierarchies and operator formalism. Nucl. Phys. B 366, 569-601 (1991)

8. Kharchev, S., Marshakov, A., Mironov, A., Morozov, A., Pakuliak, S.: Conformal matrix models as an alternative to conventional multi-matrix models. Nucl. Phys. B 404, 717-750 (1993)

9. Itzykson, C., Zuber, J.B.: The planar approximation II. J. Math. Phys. 21, 411$421(1980)$

10. Jimbo, M., Miwa, T.: Solitons and infinite dimensional lie algebras. Publ. RIMS Kyoto Univ. 19, 943-1001 (1983)

11. Hall, M.J.W.: Random quantum correlations and density operator distributions. Phys. Lett. A. 242, 123-129 (1998). arXiv:quant-ph/9802052

12. Harnad, J., Orlov, A.Y.: Fermionic construction of partition functions for two-matrix models and perturbative Schur function expansions. J. Phys. A Math. Gen. 39(28), 8783 (2006)

13. Hirota, R., Ohta, Y.: Hierarchies of coupled soliton equations. I. J. Phys. Soc. Japan 60(3), 798-809 (1991)

14. Kac, V., van de Leur, J.: The $n$-component $K P$ hierarchy and representation theory. J. Math. Phys. 44, 3245-3293 (2003)

15. Kac, V., van de Leur, J.: The geometry of spinors and the multicomponent BKP and DKP hierarchies. CRM Proc. Lect. Notes 14, 159-202 (1998)

16. van de Leur, J.W.: Matrix integrals and geometry of spinors. J. Nonlinear Math. Phys. 8, 288-311 (2001)

17. van de Leur, J.W., Yu. Orlov, A.: Random turn walk on a half line with creation of particles at the origin. Phys. Lett. A. pp. 2675-2681 (2009). arXiv:0801.0066 [math-ph]

18. van de Leur, J.W., Yu. Orlov, A.: Multicomponent $B K P$ tau function and multicomponent $K P$ tau function. Russ. J. Theor. Math. Phys. (2015)

19. Macdonald, I.G.: Symmetric functions and hall polynomials. Clarendon Press, Oxford (1995)

20. Mehta, M.L.: Random matrices, 3rd edn. Elsevier, Academic, San Diego (2004)

21. Mineev-Weinstein, M., Wiegmann, P., Zabrodin, A.: Integrable structure of interface dynamics. Phys. Rev. Lett. 84, 5106-5109 (2000)

22. Mironov, A., Morozov, A., Semenoff, G.: Unitary matrix integrals in the framework of the generalized kontsevich model. Int. J. Mod. Phys. A. 11, 5031-5080 (1996)

23. Osipov, V.A., Sommers, H.-J., Zyczkowski, K.: J. Phys. A Math. Theor. 43, 055302 (2010). arXiv:0909.5094

24. Yu. Orlov, A.: Deformed Ginibre ensembles and integrable systems. Phys. Lett. A. 378, 319-328 (2014)

25. Yu. Orlov, A., Shiota, T., Takasaki, K.: Pfaffian structures and certain solutions to BKP hierarchies I. Sums over partitions. arXiv:1201.4518 [math-ph]

26. Yu. Orlov, A., Shiota, T., Takasaki, K.: Pfaffian structures and certain solutions to BKP hierarchies II. Multiple integrals. Russ. J. Theor. Math. Phys. (2015)

27. Sato, M., Sato, Y.: Soliton equations as dynamical systems on infinite-dimensional Grassmann manifold. Nonlinear partial differential equations in applied science (Tokyo, 1982), vol. 81, pp. 259-271. North-Holland Math. Stud., North-Holland (1983)

28. Takasaki, K.: Initial value problem for the Toda lattice hierarchy. Adv. Stud. Pure Math. 4, 139-163 (1984) 
29. Takasaki, K.: Auxilary linear problem, difference Fay identities and dispersionless limit of Pfaff-Toda hierarchy. SIGMA. 5, 109 (2009). arXiv:0908.3569 [nlin]

30. Takebe, T.: Representation theoretical meaning of initial value problem for the Toda lattice hierarchy I. LMP 21, 77-84 (1991)

31. Takebe, T.: Representation theoretical meaning of initial value problem for the Toda lattice hierarchy II. Publ. RIMS Kyoto Univ. 27, 491-503 (1991)

32. Ueno, K., Takasaki, K.: Toda lattice hierarchy. Adv. Stud. Pure Math. 4, 1-95 (1984)

33. Ya. Vilenkin, N., Klimyk, A.U.: Representation of lie groups and special functions. Volume 3: classical and quantum groups and special functions. Kluwer Academic Publishers (1992)

34. You, Y.: Polynomial solutions of the BKP hierarchy and projective representations of symmetric groups. Infinite-dimensional Lie algebras and groups (Luminy-Marseille, 1988), Adv. Ser. Math. Phys., vol. 7, pp. 449-464. World Sci. Publ., Teaneck (1989)

35. Zinn-Justin, P.: HCIZ integral and 2D Toda lattice hierarchy. Nucl. Phys. B. 634(3), 417-432 (2002)

36. Zinn-Justin, P., Zuber, J.B.: On some integrals over the $\mathrm{U}(\mathrm{N})$ unitary group and their large limit. arXiv:math-ph/0209019 